\title{
FY13 LLNL OMEGA Experimental Programs
}

R. F. Heeter, K. B. Fournier, K. Baker, G. V. Brown, D. Casey, P. Celliers, D. Fratanduono, J. Hawreliak, C. Huntington, A. Lazicki, T. Ma, M. May, A. Moore, A. Pak, H. S. Park, R. Patterson, F. Perez, Y. Ping, C. Plechaty, H. Rinderknecht, J. S. Ross, J. R. Rygg, V. Smalyuk, R. Tommasini, C. Wehrenberg, A. Zylstra, G. Collins, O. L. Landen, A. Wan, W. Hsing

October 22, 2013 
This document was prepared as an account of work sponsored by an agency of the United States government. Neither the United States government nor Lawrence Livermore National Security, LLC, nor any of their employees makes any warranty, expressed or implied, or assumes any legal liability or responsibility for the accuracy, completeness, or usefulness of any information, apparatus, product, or process disclosed, or represents that its use would not infringe privately owned rights. Reference herein to any specific commercial product, process, or service by trade name, trademark, manufacturer, or otherwise does not necessarily constitute or imply its endorsement, recommendation, or favoring by the United States government or Lawrence Livermore National Security, LLC. The views and opinions of authors expressed herein do not necessarily state or reflect those of the United States government or Lawrence Livermore National Security, LLC, and shall not be used for advertising or product endorsement purposes.

This work performed under the auspices of the U.S. Department of Energy by Lawrence Livermore National Laboratory under Contract DE-AC52-07NA27344. 


\section{FY13 LLNL OMEGA Experimental Programs}

R. HEETER, K. FOURNIER, K. BAKER, G. BROWN, D. CASEY, P. CELLIERS, D. FRATANDUONO, J. HAWRELIAK, C. HUNTINGTON, A. LAZICKI, T. MA, M. MAY, A. MOORE, A. PAK, H.-S. PARK, R. PATTERSON, F. PEREZ, Y. PING, C. PLECHATY, H. RINDERKNECHT (MIT), J. ROSS, R. RYGG, V. SMALYUK, R. TOMMASINI, C. WEHRENBERG, A. ZYLSTRA (MIT), G. COLLINS, O. LANDEN, A. WAN, AND W. HSING

In FY13, LLNL's High-Energy-Density Physics (HED) and Indirect Drive Inertial Confinement Fusion (ICF-ID) programs conducted several campaigns on the OMEGA laser system and on the EP laser system, as well as campaigns that used the OMEGA and EP beams jointly. Overall LLNL led 290 target shots involving the OMEGA laser system and 158 target shots involving the EP laser system. Approximately $34 \%$ of the total number of shots ( 93 OMEGA shots, 58 EP shots) shots supported the Indirect Drive Inertial Confinement Fusion Campaign (ICF-ID). The remaining 66\% (197 OMEGA shots and 100 EP shots) were dedicated to experiments for High-Energy-Density Physics (HED).

This work performed under the auspices of the U.S. Department of Energy by Lawrence Livermore National Laboratory under Contract DE-AC52-07NA27344. 


\section{Table of Contents:}

\section{INDIRECT DRIVE INERTIAL CONFINEMENT FUSION CAMPAIGNS}

1. A New Platform for Absolute Equation-of-State Measurements for ICF (PI: D. Fratanduono) 3

2. VISAR Measurements of the EOS of Boron Carbide (PI: D. Fratanduono)

3. Shock Release Isentrope Measurements of ICF-Relevant Materials (PI: D. Fratanduono)

4. Thomson Scattering Measurements from Au Spheres (PI: J.S. Ross)

5. Measuring the adiabatic index of polystyrene using counter-propagating shocks and X-ray scattering (PI: A. Pak) 9

6. Angularly Resolved X-ray Thomson Scattering Measurements of Shock-Compressed Aluminum

(PI: L. Fletcher, LLNL/UCB Collaboration)

7. Enhanced Bremsstrahlung Backlighters (PI: R. Tommasini) 14

8. Hohlraum Drive Spectroscopy (PI: G. Brown and M. Schneider) 15

9. Ablator Opacity Measurements (PI: R. Heeter) 16

10. Hohlraum Wall-Plasma Emissivity Measurements (PI: R. Heeter) 19

11. DD and D3He Yield Anomalies vs. D:3He Fuel Ratio in Indirect Drive Exploding Pushers (PI: H. Rinderknecht, LLNL/MIT Collaboration)

12. Platform Development for Measuring Charged-Particle Stopping in Warm Dense Plasmas (PI: A. Zylstra, LLNL/MIT Collaboration)

\section{Material Dynamics and Strength}

A. Tantalum Rayleigh-Taylor Experiments (PI C. Plechaty) 22

B. Iron Rayleigh-Taylor Experiments (PI C. Huntington) 24

C. Long-Pulse Silver Backlighter Development (PI: C. Huntington) 25

D. Material Recovery Experiment (PI: M. May) 26

E. Diffraction Studies on Shocked Tantalum (PI: C. Wehrenberg) 27

F. Classical Rayleigh-Taylor Experiment 29

II. Equation-of-State $\quad 3$

A. Demonstration of Single-Shot XAFS Measurements on Ramp-Compressed Ta (PI: Y. Ping) 30

B. Tin Melting and Recrystallization (PI: A. Lazicki) 31

C. Tantalum X-Ray Diffraction with Ramp Compression (PI: A. Lazicki) 32

D. Development of a Soller Slit Diagnostic for Dynamic Diffraction Studies (PI: J. Hawreliak) 33

E. Radiographic Equation of State Measurements of Shocked Foams (PI: J. Hawreliak) 34

F. High Energy X-ray Diffraction Development (PI: J. Hawreliak) 35

G. Lithium Hydride Equation of State (PI: A. Lazicki) 36

H. Tantalum Equation of State (PI: D. Fratanduono) 36

$\begin{array}{ll}\text { III. Radiation Transport and Opacity } & 37\end{array}$

$\begin{array}{ll}\text { A. Heated Wall Radiation Transport (PI: K. Baker) } & 37\end{array}$

IV. Hydrodynamics $\quad 38$

A. Toto - Radiography Development for NIF Hydrodynamics Experiments (PI: V. Smalyuk) 38

B. Copper Foam Shock Breakout Measurements (PI: A. Moore) 39

C. Short-pulse, UV backlighting development for NIF (PI: D. Martinez) 40

D. X-ray Area Backlighter Development (PI: K. Baker) 41

V. Burn Physics $\quad 42$

High-resolution measurements of velocity nonuniformities in a boron carbide ablator (PI: P. Celliers) 42

Ablator Physics: Tests of Beryllium Capsules (PI: D. Casey) 45

VI. X-Ray Source Development and Applications 46

A. Solar Cell Electrostatic Discharge (PI: R. Patterson) 46

B. X-Ray Source Development with Nanostructured Materials (PI: R. Patterson) 49 


\section{Indirect Drive Inertial Confinement Fusion Experiments}

\section{A New Platform for Absolute Equation of State Measurements (PI: D. Fratanduono)}

A radiographic equation-of-state (EOS) platform was developed using OMEGA-EP in order to be used at the NIF facility. The concern regarding traditional VISAR-based EOS platforms is that at high pressures ( $>5000 \mathrm{GPa}$ ), optical windows (i.e. Quartz and LiF) would become opaque due to x-ray preheat or radiative shock fronts. As a result, at such extreme pressures the VISAR diagnostic would no longer be useful to perform EOS measurements. In addition, VISAR EOS measurements at NIF and OMEGA are not absolute, as they rely upon a standard reference. Therefore, a new experimental platform is required to perform high-pressure absolute Hugoniot measurements.

Radiography experiments have been conducted in order to measure the shock density and shock velocity at high pressure. The purpose of these experiments was to radiograph a shock front in a low-z sample $(\mathrm{CH})$ as shown in Figure (1). The radiographs are used to determine the shock velocity and the shock density of the compressed materials. ABSEOS-13A-EP was successful in demonstrating the platform. We were able to determine the EOS of the $\mathrm{CH}$ sample. Measurements are in agreement with previous experiments performed by M.A. Barrios using VISAR as shown in Figure (2). The platform design was improved for a second campaign, ABSEOS-13A-EP, to reduce high background levels in the emission and to resolve parallax issues. Both issues were resolved in ABSEOS-13B-EP, producing better quality data. Future platform development would be to conduct experiments on OMEGA, using halfraum drives, since the OMEGA-EP experiments were direct drive.

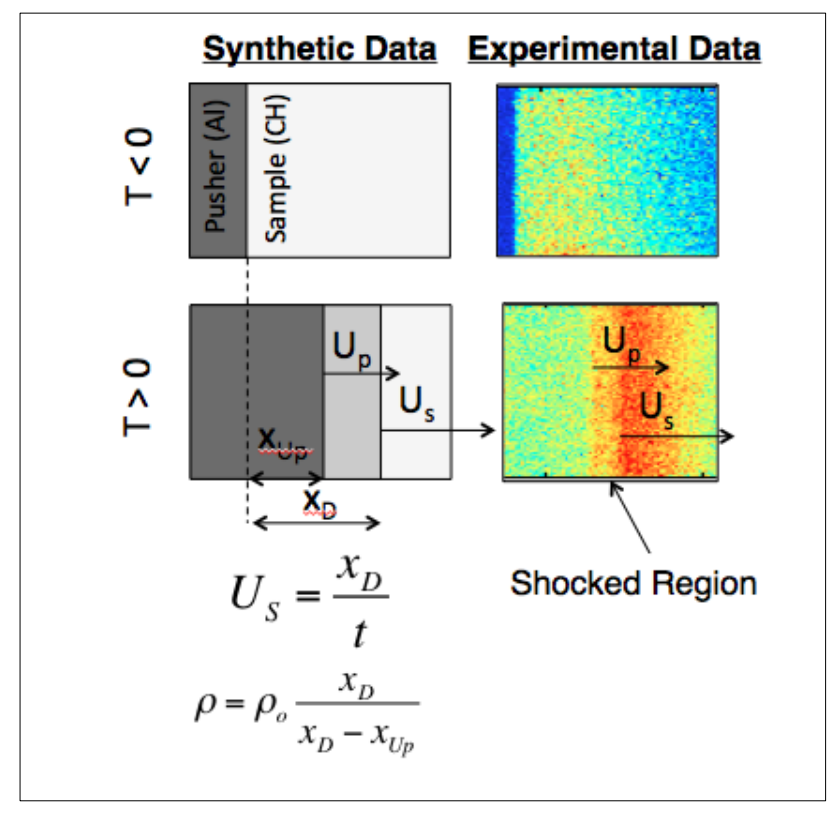

Figure 1: Synthetic and experimental data illustrating the radiography technique to perform Hugoniot experiments. 
Figure 2: Comparison of radiography measurements with experimental fit to $\mathrm{CH}$ data from the thesis of M.A. Barrios.

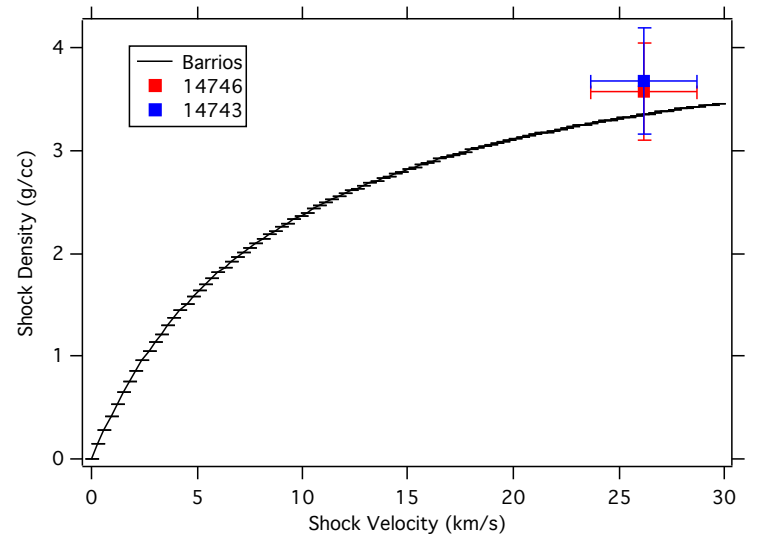




\section{VISAR Measurements of the EOS of Boron Carbide (PI: D. Fratanduono)}

The ICF campaign has begun looking into alternate ablators (i.e. Diamond, $\mathrm{B}_{4} \mathrm{C}$ and $\mathrm{SiC}$ ). As a result, a campaign to measure the Hugoniot of $\mathrm{B}_{4} \mathrm{C}$ was conducted. Previous Hugoniot measurements of $\mathrm{B}_{4} \mathrm{C}$ were limited to $\sim 200 \mathrm{GPa}$ due to the limiting pressure range using a two-stage gas gun. Experiments were conducted at the OMEGA laser facility to extend the pressure range to $800 \mathrm{GPa}$. Twelve experiments were conducted which measured the Hugoniot from $\sim 250 \mathrm{GPa}$ to $200 \mathrm{GPa}$. Experimental data is shown in Figure (3). Measurements are consistent with lower pressure data. A slight disagreement with LEOS table 2120 and Sesame table 7082 is observed. These data will be used to generate a new EOS model that will assist in hydrocode simulations and future experimental design for ICF.

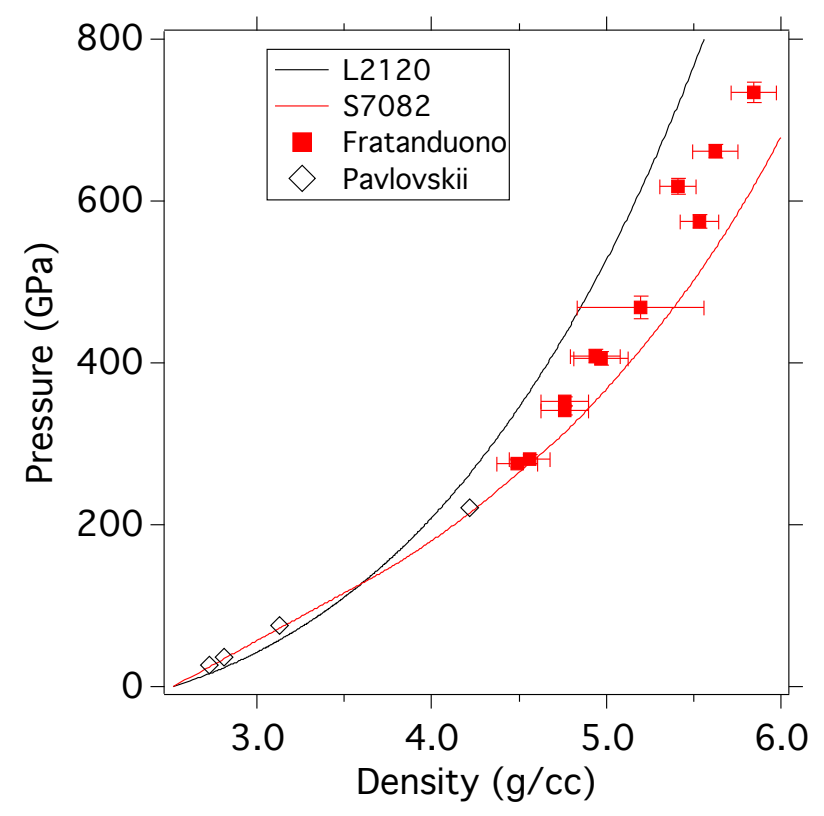

Figure 3: B4C Hugoniot measurements (as red squares) from B4C-EOS-13A campaign. Measurements are compared with low-pressure Pavlovskii data, LEOS table 2120 and Sesame table 7082. 


\section{Shock Release Isentrope Measurements of ICF Relevant Materials (PI: D. Fratanduono)}

The goal of this campaign was to demonstrate the feasibility of previous experimental designs using thin silicon nitride films $(150 \mathrm{~nm})$ with a low-density methane gas $(0.6 \mathrm{mg} / \mathrm{cc})$ present on both sides of the film simulating the $\mathrm{D}_{2}$ vapor that would be present on a NIF capsule experiment. Previously, the thin films acted as a boundary between methane and vacuum. The concern was that the methane gas behind the silicon nitride would become opaque to the VISAR when the shock passes through the silicon nitride film. Experimental results indicated that the methane gas is transparent to the VISAR probe and the silicon nitride velocity was observed. Results also indicated that the presence of gas on both sides of the film helped to tamp the nitride and prevent it from breaking during the experiment. Results continue to indicate that the LEOS tables are over predicting the velocity of the release isentropes.

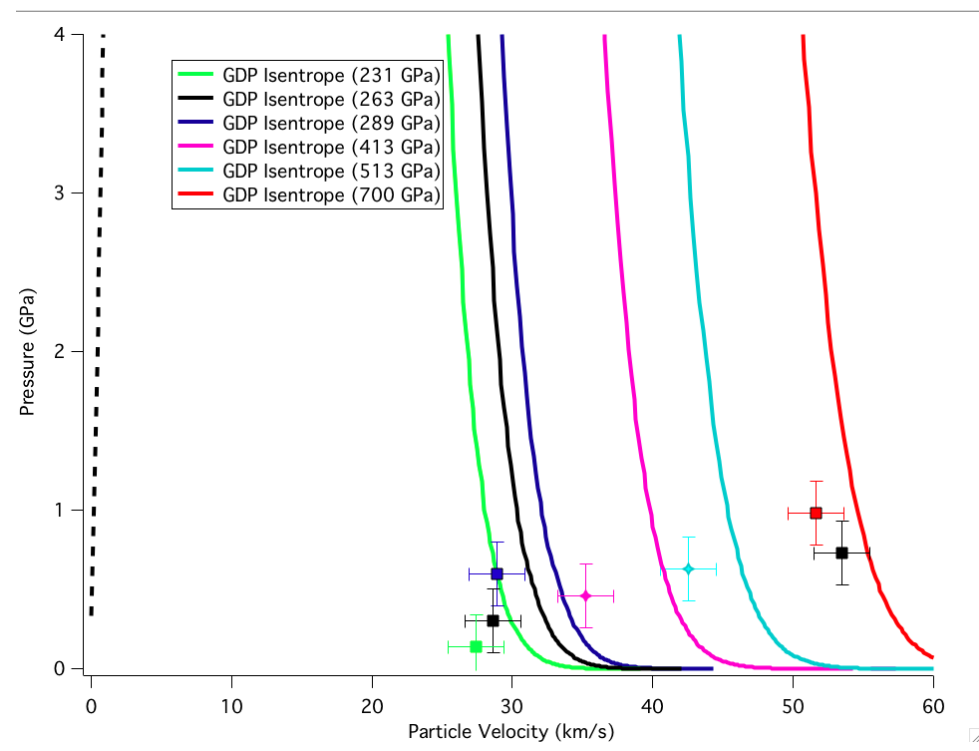

Figure 4: Experimental measurements are shown as squares and are colored coded with release isentropes from LEOS table for CH. Measurements indicate that the LEOS models are over predicted the release velocity. 


\section{Thomson scattering measurements from Au Spheres (PI: J.S. Ross)}

with E. Dewald (LLNL), M. Rosen (LLNL), D. Froula (LLE)

In the AuSphere-13A campaign we have performed high- $Z$ sphere experiments illuminated in direct drive geometry to investigate atomic physics models and radiative properties of the laser spot plasma relevant to ICF indirect drive ignition hohlraum plasmas. $1 \mathrm{~mm}$ Au spheres are irradiated using SG4 laser phase plates to deliver uniform illumination of the target with 3-30 kJ of laser energy at intensities of $0.1,0.5$, and $1 \times 10^{15} \mathrm{~W} / \mathrm{cm}^{2}$, similar to the intensities found in NIC hohlraums. The $4 \omega$ Thomson scattering probe beam was aligned at various radial locations ranging from 100 to 300 microns from the target surface, and used to characterize the low-density plasma blowoff. All of the laser beams used a 1ns square laser pulse. An example of the Thomson scattering ion feature data is shown in Figure 5, taken at 200 microns from the target surface.

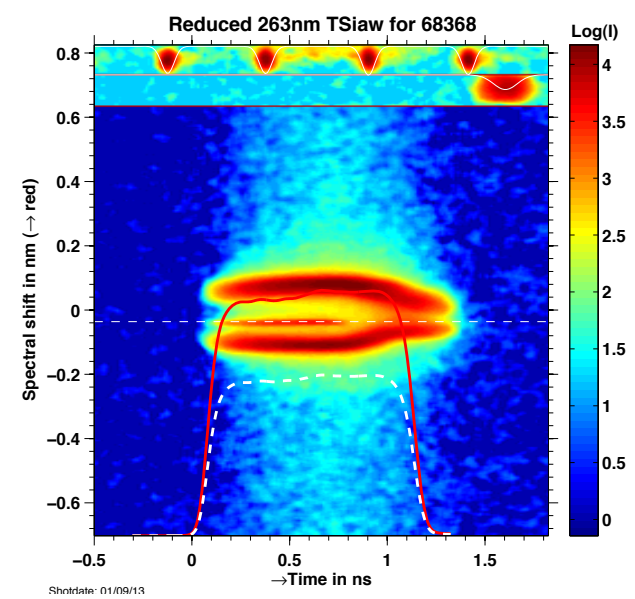

Figure 5: Thomson scattering data from 200 microns off the surface of the Au sphere irradiated at an intensity of $5 \times 10^{14} \mathrm{~W} / \mathrm{cm}^{2}$.

The electron temperature and density, the plasma flow velocity, and the average ionization state are measured by fitting the theoretical Thomson scattering form factor to the observed data. The measured data is then compared to post-shot simulations with different atomic physic and electron transport models. The different models predict different electron temperatures for the experimental conditions and are shown in Figure 6. 
a)

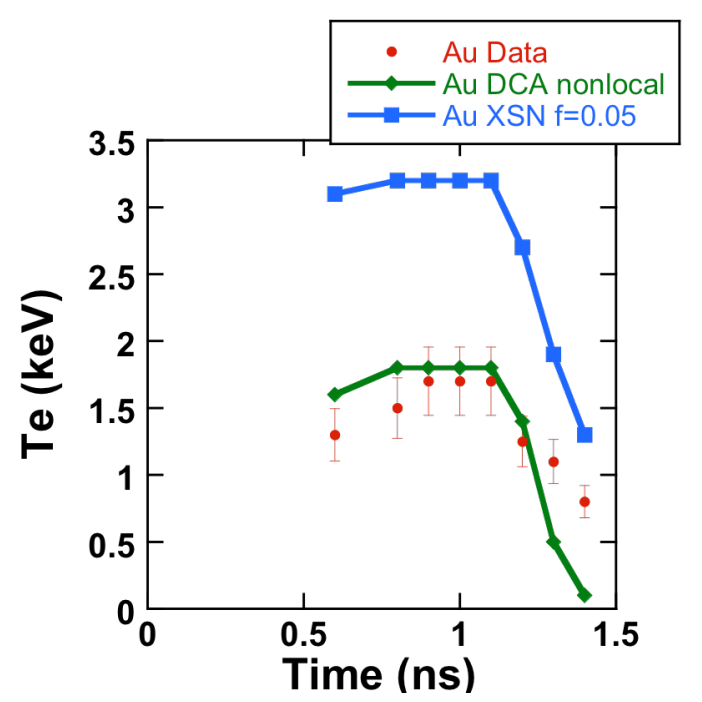

b)

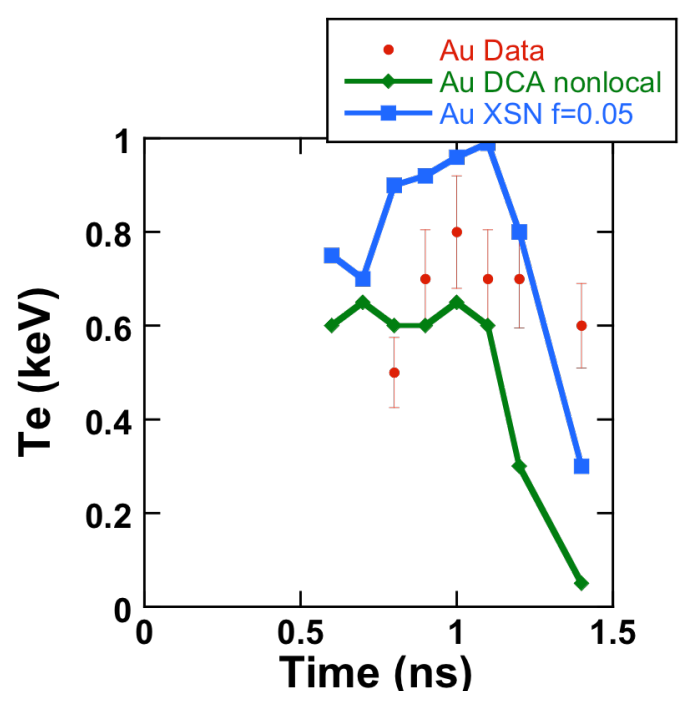

Figure 6: The measured electron temperature (red circles) is compared to post-shot simulations using the DCA nonlocal model (green line) and the XSN flux limited model (blue line) for intensities of a) $5 \times 10^{14} \mathrm{~W} / \mathrm{cm}^{2}$ and b) $1 \times 10^{14}$ $\mathrm{W} / \mathrm{cm}^{2}$ at a radial location of 200 microns.

The simulation with the DCA nonlocal model most closely reproduces the temperatures measured during the experiment. The XSN model with a flux limited transport model using a flux limiter of 0.05 , previously used to simulate high-Z hohlraums, predicts an electron temperature significantly higher than measured. Additional measurements at different radial locations and different intensity appear most consistent with an intermediate model with a temperature between that predicated by the XSN and DCA models. Further data analysis is underway to compare Thomson scattering data, DANTE absolute x-ray flux measurements and X-ray spectroscopy to simulations in our effort to validate atomic physics models employed in simulations. 


\section{Measuring the adiabatic index of polystyrene using counter-propagating shocks and x-ray Thomson scattering (PI: A. Pak)}

With T. Ma, L. Fletcher, T. Doppner, S.H. Glenzer and O.L. Landen

Understanding the equation of state and opacity of polystyrene, a material similar to the NIF ablator, at densities of a few $\mathrm{g} / \mathrm{cc}$ and at temperatures of several $\mathrm{eV}$, is of interest as it can inform the design and performance of current implosion experiments. In the $\mathrm{CH}$-HeatCap campaign at Omega EP the electron density, temperature and charge state of shocked polystyrene $(\mathrm{CH})$ was directly measured with x-ray Thomson scattering (XRTS). The goal of these campaigns was to test the hypothesis that the continuum lowering effect would increase the charge state of $\mathrm{CH}$ as the compression of the material is increased. Understanding in detail the charge state of $\mathrm{CH}$ could inform opacity models that are used to calculate the ablation dynamics of NIF implosions.

Figure XX a) shows the experimental setup. Here beams 2 and 3 were used to drive a shock in the $\mathrm{CH}$ sample. The strength of the shock, and thus the expected post shock density, was varied from shot to shot by using three different drive intensities of $5 \mathrm{e} 12,1.5 \mathrm{e} 13$ and $2 \mathrm{e} 14 \mathrm{~W} / \mathrm{cm}^{2}$. The shocked state was probed using the zinc He- $\alpha$ line at an energy of $9 \mathrm{keV}$. This line was produced by irradiating a zinc foil, placed $1.6 \mathrm{~mm}$ away from the $\mathrm{CH}$ sample, with beams 1 and 4 at an intensity of $\sim 1 \mathrm{e} 15 \mathrm{~W} / \mathrm{cm}^{2}$. $\mathrm{X}$-rays that were scattered at an angle of $154^{\circ}$ with respect to the incident angle were collected by a curved HOPG crystal placed in the ZSPEC spectrometer. The HOPG crystal spectrally disperses the transversely focused scattered signal onto a framing camera detector that has a integration time of 250300 ps.

Figure XX b) compares the spectral intensity measured for 3 different shots, undriven, driven at $\mathrm{I}=5 \mathrm{e} 12 \mathrm{~W} / \mathrm{cm}^{2}$, and at $\mathrm{I}=2 \mathrm{e} 14 \mathrm{~W} / \mathrm{cm}^{2}$. Detailed fits of the scattered spectrum using the current description of the bound free scattering component have been unable to accurately model the data. A revised code that incorporates a more accurate model of the bound free spectrum has been developed and will be applied to this data in the future. Qualitatively, the increase in charge state as the density of the shock state is increased is believed to have been observed. This is shown in Fig. XX c) where the inelastic components of the 3 spectra have been overlaid. As the drive intensity is increased, the spectral width of the inelastic feature is observed to increase on the higher energy side, while decreasing on the lower energy side. It is believed that the decrease in spectral width on the low energy side is due to the decreased L-shell contribution in the bound free spectra, while the broadening on the high energy side is due to an increase in the electron density.

The initial results of Heat Capacity campaign appear of sufficient quality to determine if continuum lowering is occurring and how the final charge state, density and temperature vary with the magnitude of the drive intensity. 

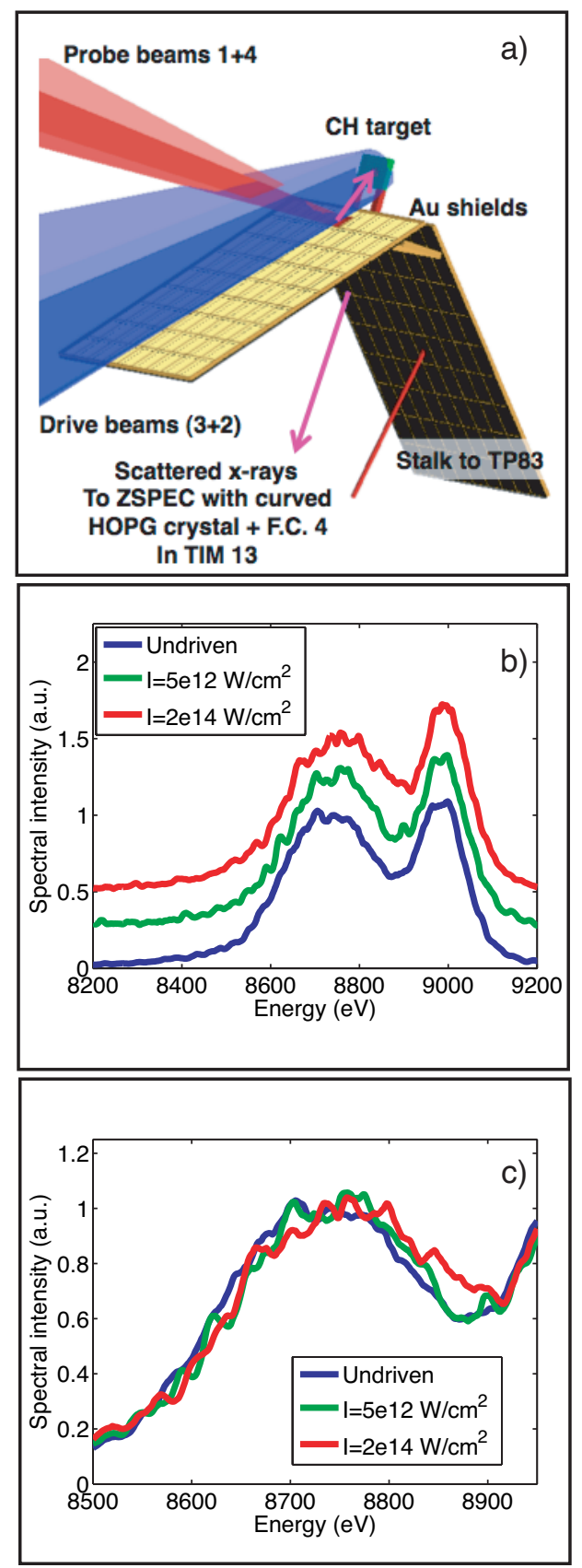

Figure XX a) Experimental setup. b) Scattered spectrum observed at three different drive conditions. Here the signals have been normalized and offset from each other. c) Overlay of the normalized inelastic scattered feature where the decrease in the width on the low energy side as the drive intensity is increased may be an indication of continuum lowering. 


\section{Angularly Resolved X-ray Thomson Scattering Measurements of Shock-Compressed Aluminum (P.I.: L. Fletcher, University of California - Berkeley)}

with T. Ma, A. Pak, H. J. Lee, T. Döppner, C. Fortmann, O. L. Landen (LLNL), S. H. Glenzer (Stanford), and R. Falcone (UCB)

We have directly demonstrated the ability to measure the ion-ion correlation peak using simultaneous angularly, temporally, and spectrally resolved x-ray scattering measurements in laser-driven shockcompressed aluminum. Molybdenum He-alpha (17.9 keV) x-rays have been used to probe Al foils that are compressed using both single and double (counter propagating) shocks in a forward, small angle, scattering platform with very high angular precision.

In our study, $125 \mu \mathrm{m}$ thick and $200 \mu \mathrm{m}$ thick Al targets were compressed 3x and 6x the solid density using $1 \mathrm{~ns}$ pulses with a total laser energy of $4.5 \mathrm{~kJ}$ and $9 \mathrm{~kJ}$ respectively. A total drive intensity of $3 \times 10^{14} \mathrm{~W} / \mathrm{cm}^{2}$ on each irradiated aluminum surface was used to drive shocks into the sample. For backlighting, $7 \times 10^{14} \mathrm{~W} / \mathrm{cm}^{2}$ incident on a thin Mo foil was used to generate Mo He- $\alpha$ x-rays at $17.9 \mathrm{keV}$ to probe the compressed targets. Figure $2 \mathrm{~b}$ demonstrates a shift in a well-pronounced peak in the static structure factor at wave numbers of $\mathrm{k}=3.5 \AA^{-1}$ and $4.5 \AA^{-1}$ corresponding to higher compression material states.

The magnitude of this correlation peak is in good agreement with calculations that use a potential with strong short range repulsion (SRR). The results will show that the elastic $\mathrm{x}$-ray scattering amplitude, angularly resolved, shifts to higher wave number of approximately $\Delta \mathrm{k}=1 \times 10^{-10} \mathrm{~m}^{-1}$ with increasing density. This presents a new scattering diagnostic to fully characterize states of matter at densities and temperatures that have not been previously accessible. 
(a)

MCP detector (curved HOPG)

9 probe beams

(9.5 kJ, 1ns)

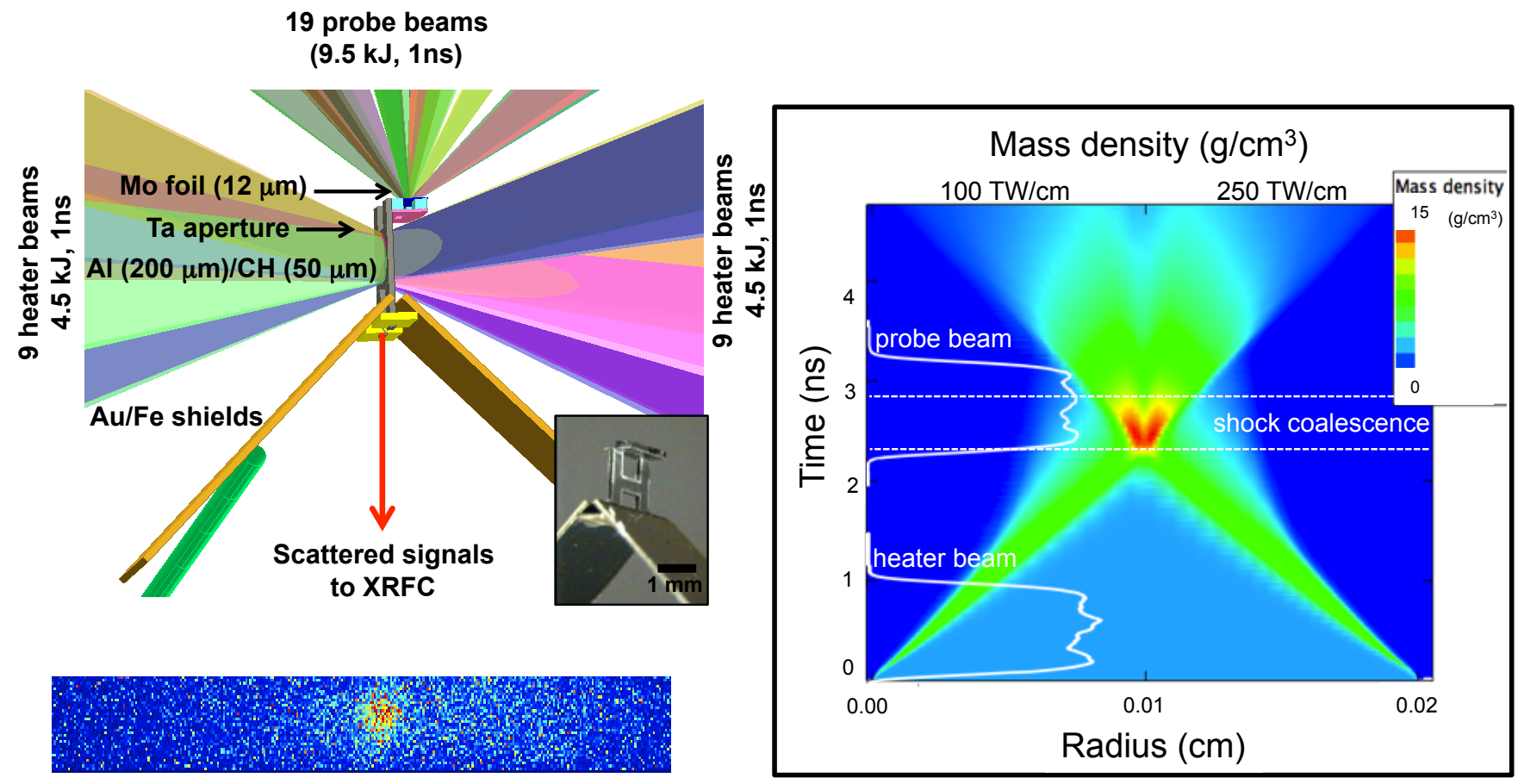

(b)

Figure YY1 - The experimental setup for HiZShk-13A; a.) $200 \mu \mathrm{m}$ thick Al target (double-shock configuration) and beam configuration, along with a photo of Al scattering target, and gated XRFCMCP detector data; b.) 2-D Helios simulation of the mass density is shown as a function of $\mathrm{Al}$ foil radius, and input pulse shape dimensions (TW and time duration). 
(a)

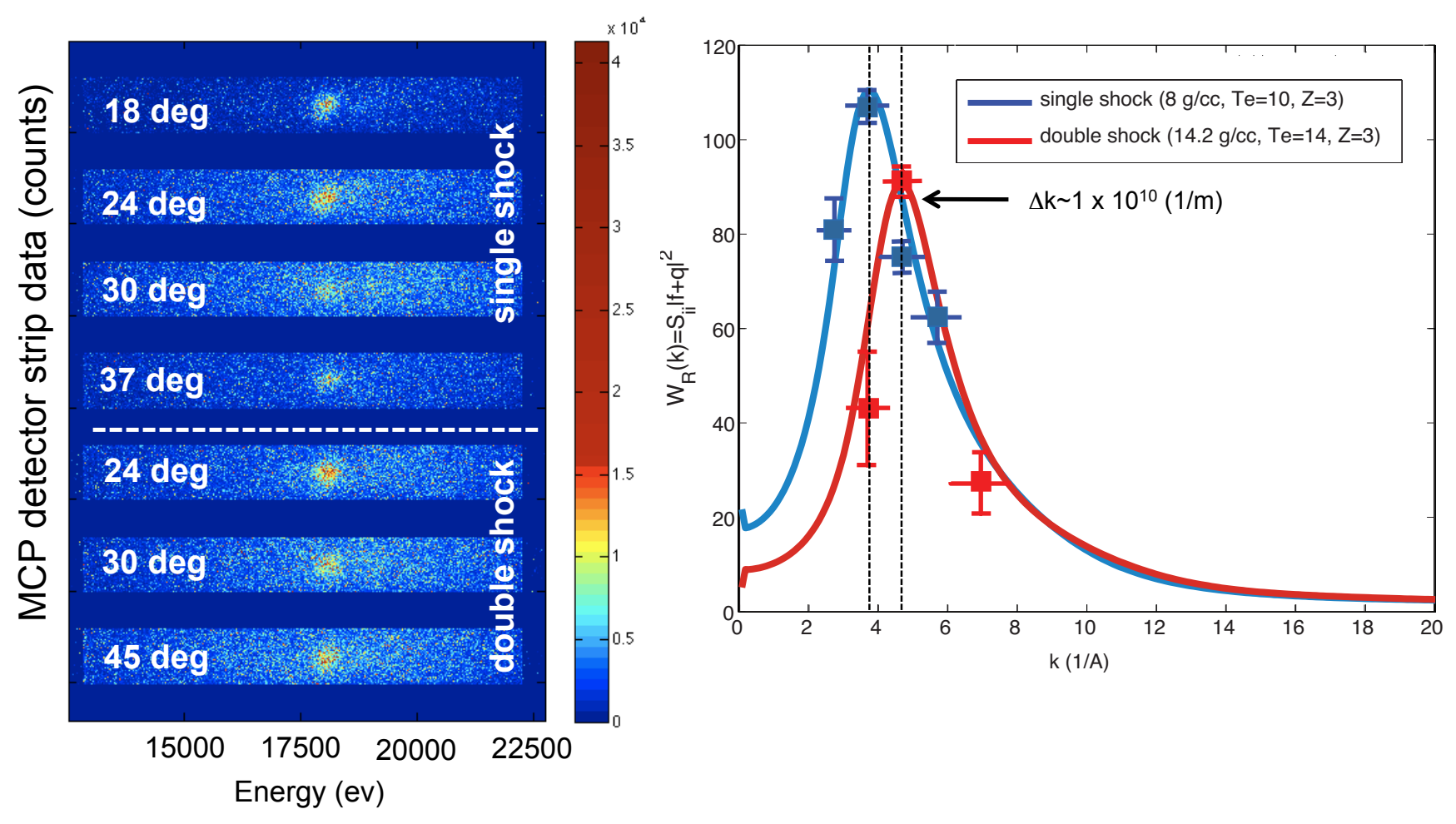

Figure YY2 - a.) Raw data of scattered spectrally resolved XRTS signals for single and double shock configurations; b.) Preliminary data analysis shows the total integrated signal (corrected for experimental geometry) of the scattering data as a function of $\mathrm{k}$ follows the trends predicted by the SRR models using the frequency-integrated, quasielastic contribution to the dynamic structure factor of single shocked $(\rho=8 \mathrm{~g} / \mathrm{cc})$ and double shocked $(\rho=14 \mathrm{~g} / \mathrm{cc})$ Aluminum. 


\section{Enhanced Bremsstrahlung backlighters (PI: R. Tommasini)}

The motivation of the UV-CR-BL-13A campaign was to test and characterize enhanced broadband $\mathrm{x}$-ray sources emitting around and above $75 \mathrm{keV}$, to be used in the Compton Radiography of ICF targets on the NIF. In FY09 we characterized Au micro-wire backlighters irradiated by 10ps-long, OMEGA EP pulses. In FY10 we characterized $\mathrm{Au}$ micro-wire backlighters irradiated by 100ps-long, OMEGA EP pulses $(1 \omega)$ and OMEGA pulses $(3 \omega)$, with the primary goal of measuring the effect of longer-pulse generated backlighter with respect to the source size. In FY13 we characterized Au micro-wire backlighters irradiated by $1 \mathrm{~ns}$ OMEGA pulses $(3 \omega)$, and compared the performance of wires with and without $\mathrm{CH}$ wedges (see Fig.ZZ).

The $30 \mu \mathrm{m} \mathrm{Au} \mathrm{micro} \mathrm{wires} \mathrm{are} \mathrm{arranged} \mathrm{in} \mathrm{a} \mathrm{point-}$ projection end-on geometry, and are $\sim 500 \mu \mathrm{m}$ long. They were mounted on a $\mathrm{CH}$ slab and, in about half of the performed shots, they were sitting inside a $\mathrm{CH}$ wedge, with $30^{\circ}$ half angle. The Bremsstrahlung radiation was generated by irradiating the $\mathrm{Au}$ wires with $1 \mathrm{~ns}$ square pulses. The energy on target was changed between 500J and $2500 \mathrm{~J}$ by changing the number of incident beams, from one to five.

As radiography samples we used solid $\mathrm{W}$ spheres with diameters of $200 \mu \mathrm{m}$ located at a distance of $10 \mathrm{~mm}$ from the backlighters.

In order to record the radiographs and measure the time integrated backlighter spectra, we used the Compton Radiography Snout (CRS). A combination of high- and
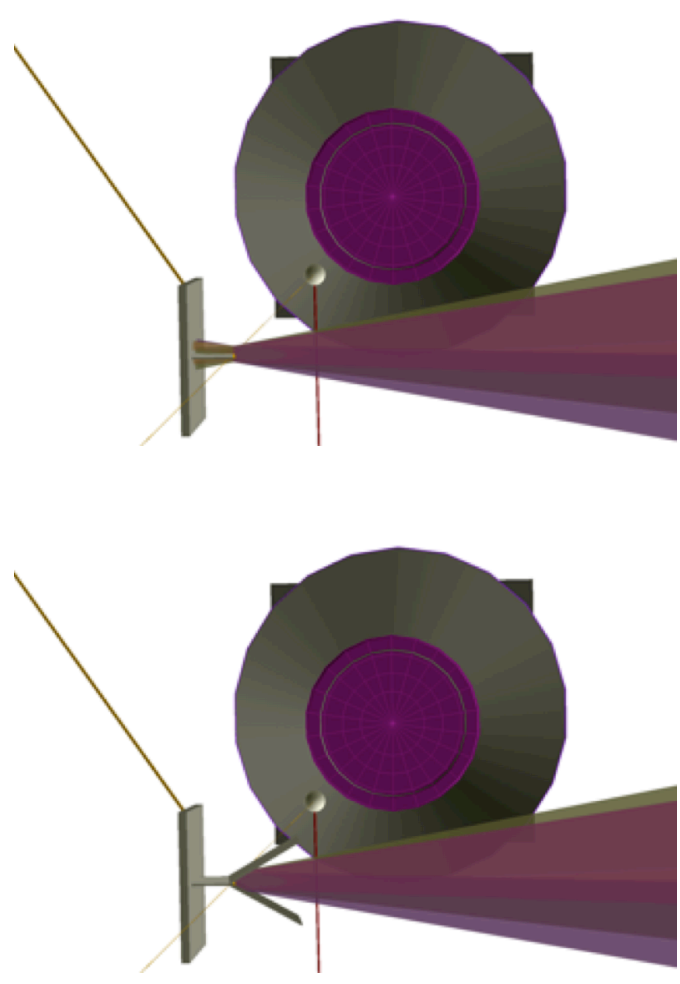

Fig. ZZ: Backlighter geometry used for the experiments. Top: bare Au wire. Bottom: Au wire inside $\mathrm{CH}$ wedge. low-Z filters was used inside the snout to restrict the backlit photon energies to about and above $75 \mathrm{keV}$. As a detector we used a Fuji BAS imaging plate at $400 \mathrm{~mm}$ distance from the backlighter. The NTD diagnostic was used to record the time resolved emission from the backlighter. The data show an increase in the peak of the time resolved backlighter signal by $\sim 4 \mathrm{x}$ and in the total time integrated signal by $\sim 2 \mathrm{x}$ when using wire in $\mathrm{CH}$ wedge. These values are very close to what predicted by simulations. The analysis of the radiographs of the $\mathrm{W}$ sphere allows the reconstruction of the source size of the backlights. We measured $\sim 30 \mathrm{um}$ for the $\mathrm{Au}$ wire in $\mathrm{CH}$ wedge vs. $45 \mathrm{um}$ for the bare $\mathrm{Au}$ wire, which we ascribe to plasma confinement generated by the $\mathrm{CH}$ wedge. 


\title{
8. Hohlraum Drive Spectroscopy (PI: G. Brown and M. Schneider)
}

\author{
with K. Widmann, R. Heeter and J. Emig
}

The objective of the DriveSpec-13A campaign was three fold: (1) To assess what is necessary to field a fully calibrated, high resolution crystal spectrometer on NIF whose spectra can be used to refine data from the Dante diode array drive diagnostic, (2) To assess the atomic physics taking place in a hohlraum's non-LTE drive radiation field, and (3) To use the high-resolution spectra to aid in the deconvolution of the Dante spectra.

To provide benchmark high resolution spectra for models of the drive radiation for ICF, three target types were fielded. The first was a $2.250 \mathrm{~mm}$ long, $1.2 \mathrm{~mm}$ diameter, $100 \%$ open laser entrance holes (LEHs), $25 \mu \mathrm{m}$ wall gold hohlraum; the second was a gold hohlraum of the same dimensions but with the interior surfaces coated with 4-11 $\mu \mathrm{m}$ of uranium; and the third was a gold halfraum with a single $0.8 \mathrm{~mm} 100 \%$ open LEH, a length of $0.750 \mathrm{~mm}$, and closed on the side opposite the LEH. Eight of the $1.2 \mathrm{~mm}$ LEH gold targets, one 1.2 LEH uranium target, and two $0.8 \mathrm{~mm}$ LEH gold targets were shot.

Radiation from each shot was detected with several spectrometers. The main diagnostics were three high-resolution "MSpec" elliptical spectrometers, each covering a continuous bandwidth form about 1.7 to $3 \mathrm{keV}$. Two of the Mspecs recorded time integrated spectra using image plate and were on nearly the same sightline at the Dante temperature diagnostic: one at $42^{\circ}$ relative to the LEH on the H10 side of the hohlraum, i.e., on the opposite side as Dante, and one at $37^{\circ}$ on the H11 side of the hohlraum, i.e., on the opposite side of Dante. A third Mspec recorded time resolved spectra. A variable line spaced grating spectrometer (VSG) covering the $\sim 0.2$ to $1.5 \mathrm{keV}$ band was also used to record time resolved spectra.

Spectra from this campaign have already provided new insight as to the deconvolution of the Dante spectra, especially regarding the distribution of the Mband emission from highly charged gold ions. High resolution spectra of the gold Mband emission is also being compared to models used to predict the drive radiation field in NIF ICF hohlraums. Calibration measurements of the full instrument response, including filter $\mathrm{x}$-ray transmission efficiencies and Bragg crystal reflectivities have been completed. These results demonstrate technical readiness to field a fully calibrated drive spectrometer on the central channel of the NIF Dante. 


\title{
9. Ablator Opacity Measurements (PI: R. Heeter)
}

\author{
with A. Graf, G.V. Brown, C. Mauche, R.E. Marrs, and B. Wilson
}

In a followup to earlier ablator opacity measurements, improved experiments were performed to measure the transmission opacity of hot Si-doped GDP (using Omega-60) and pure Si (using Omega$\mathrm{EP}$ ) at $\mathrm{T} \approx 100 \mathrm{eV}, \rho \approx 0.1 \mathrm{~g} / \mathrm{cc}$. The Omega platform used a Be-tamped Si-doped GDP sample heated indirectly in a hohlraum. The EP platform used a CH-tamped embedded Si dot heated by 100 ps UV impulses. Both platforms used dual-axis backlighting, both face-on and edge-on, simultaneously to obtain density via sample expansion radiography, Si ionization balance data and GDP and/or Si transmission opacities via absorption spectroscopy. The GDP measurements used a high-Z backlighter to record the transmission spectrum for X-rays ranging 250 to $1600 \mathrm{eV}$, as well as from 1600 to $3000 \mathrm{eV}$. The Si measurements span the X-ray band from $1600 \mathrm{eV}$ to $3000 \mathrm{eV}$.

Compared to the prior measurements using similar opacity platforms at Omega, the sample density was increased about four-fold, and the sample uniformity was also improved, particularly for the Si on EP by using a thicker tamper on the laser-heated side. In addition, backlighter and spectrometer performance were also improved on both platforms, yielding $2 \mathrm{x}$ to $3 \mathrm{x}$ improvements in signal-to-noise of absorption and transmission measurements. The resulting data can provide constraints on opacities in conditions approaching those where capsule performance (via the Atwood Number) may depend strongly on the $\mathrm{Si}$ and C opacity (Figure XX).

The Si measurements illustrated in Figure YY can be brought into agreement with simulations for the $\mathrm{n}=1$ to $\mathrm{n}=2$ absorption features, but require a wider charge state distribution than expected in LTE, suggesting non-LTE physics or sample nonuniformities. Data for the higher-n transitions is not in especially good agreement with the opacity models. Additional Si measurements are planned for FY14. 


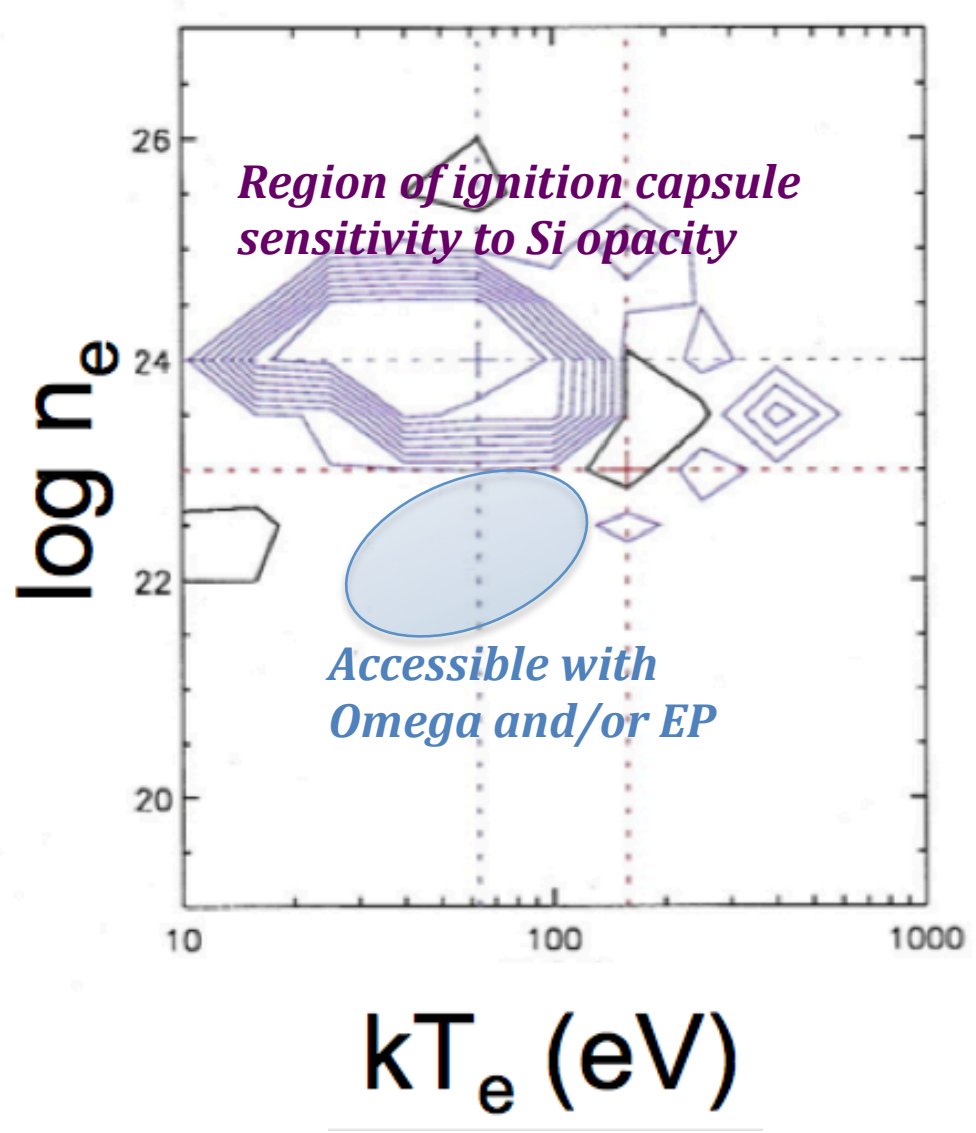

Figure XX: Using 1-D ignition capsule simulations, and varying the assumed opacity of the Si dopant in localized regions of density-temperature space by $30 \%$, it is found that the Atwood Number varies by up to $37 \%$. The sensitivity is localized for near-solid density plasmas at temperatures from $30-100 \mathrm{eV}$. The Atwood number is also sensitive to the opacity of carbon, though the specific processes and region in parameter space are different. 


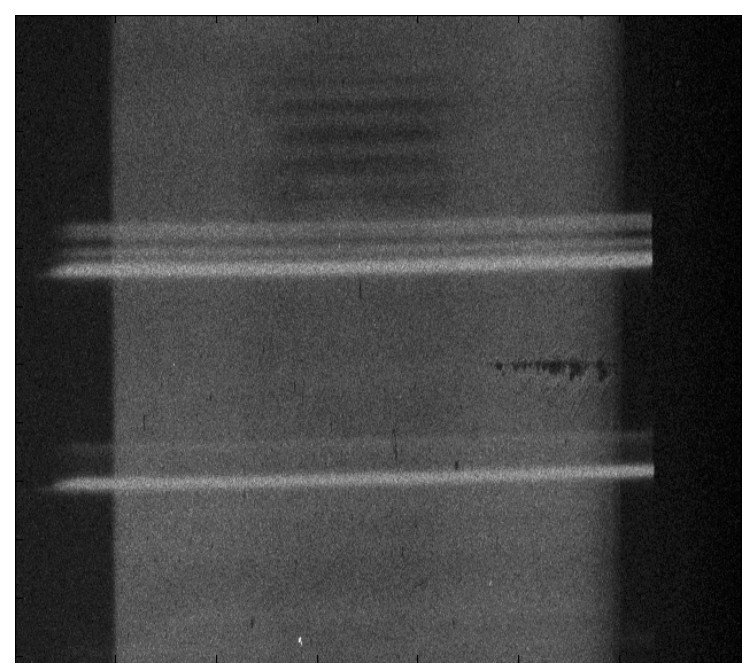

Figure YY: X-ray absorption spectrum of silicon from $\sim 1.7$ to $\sim 2.1 \mathrm{keV}$, from a plasma at $\mathrm{T} \approx 70 \mathrm{eV}, \rho$ $\approx 0.2 \mathrm{~g} / \mathrm{cm}^{3}$. Photon energy runs from top to bottom; the horizontal direction includes regions of background, sample self-emission, backlighter-only and backlit sample transmission spectra. Absorption features including $n=1$ to $n=2$ transitions, higher- $n$ features, and continuum absorption appear as a dark band running down the center of the image from top to bottom. The absorption features from multiple Lshell ion stages of Si (Be, B, C-like...) are seen as dark horizontal bands in the top center. Emission features from H-like and He-like ions of the hotter, denser Si produced prior to the short-pulse absorption measurement are also observed (horizontal white features). 


\title{
10. Hohlraum Wall-Plasma Emissivity Measurements (PI: R. Heeter)
}

\author{
with A. Graf, G.V. Brown, M.E. Foord, C. Mauche, R.E. Marrs, J.S. Ross and B. Wilson
}

Motivated by discrepancies observed in NIF hohlraum experiments, new benchmark data for the X-ray emissivity of hot gold plasmas was sought. Of particular interest were the inferred "drive deficits" on ignition capsule implosions, and observed differences in drive spectra and X-ray emission images on ViewFactor shots. The NIF data indicate a substantially different distribution of mass and temperature in the ablated gold "wall plasma" than predicted by simulations. One concern which arises is that the complex modeling of the gold itself may need better validation. This requires precision spectroscopy of uniform, near-steady-state plasmas at the relevant conditions. Prior benchmark data (Heeter, Phys. Rev. Lett. 99, 2007) was obtained at $\mathrm{n}_{\mathrm{e}}=10^{21} / \mathrm{cm}^{3}$, but on NIF the gas- and capsule-plasma filling holds back the expanding gold plasma ablated off the walls, such that the density of interest is an order of magnitude greater. In addition, alternate NIF ICF hohlraum designs incorporate a mid-Z coating (Ni or $\mathrm{Cu}$ ) to suppress the $\mathrm{Au} \mathrm{M}$-band emission, so measurements of $\mathrm{Ni}$ or $\mathrm{Cu}$ are timely as well.

In the experiment fielded late in Q4 of FY13, Be-tamped samples of $\mathrm{Au}, \mathrm{Ni}$ or comixed $\mathrm{Au}: \mathrm{Ni}$ were directly heated for $3 \mathrm{~ns}$ using a train of Omega beams. To obtain the sample density and temperature, a combination of expansion imaging, Ni K-shell spectroscopy and 4w Thomson Scattering diagnostics were applied. A sample of the Thomson data is shown in Figure XX. Using thicker tampers and lower laser drive, and imaging the sample expansion with 15-20 um resolution, plasmas with ne $>$ $2 \times 10^{22} / \mathrm{cm}^{3}$ were studied. Samples at two temperatures were produced by varying the laser intensity on different shots. Benchmark-quality absolute- and relatively-calibrated sample emission spectra were obtained from 250-3000 eV using gated, space resolved grating and crystal spectrometers and the Dante multichannel filtered diode array.
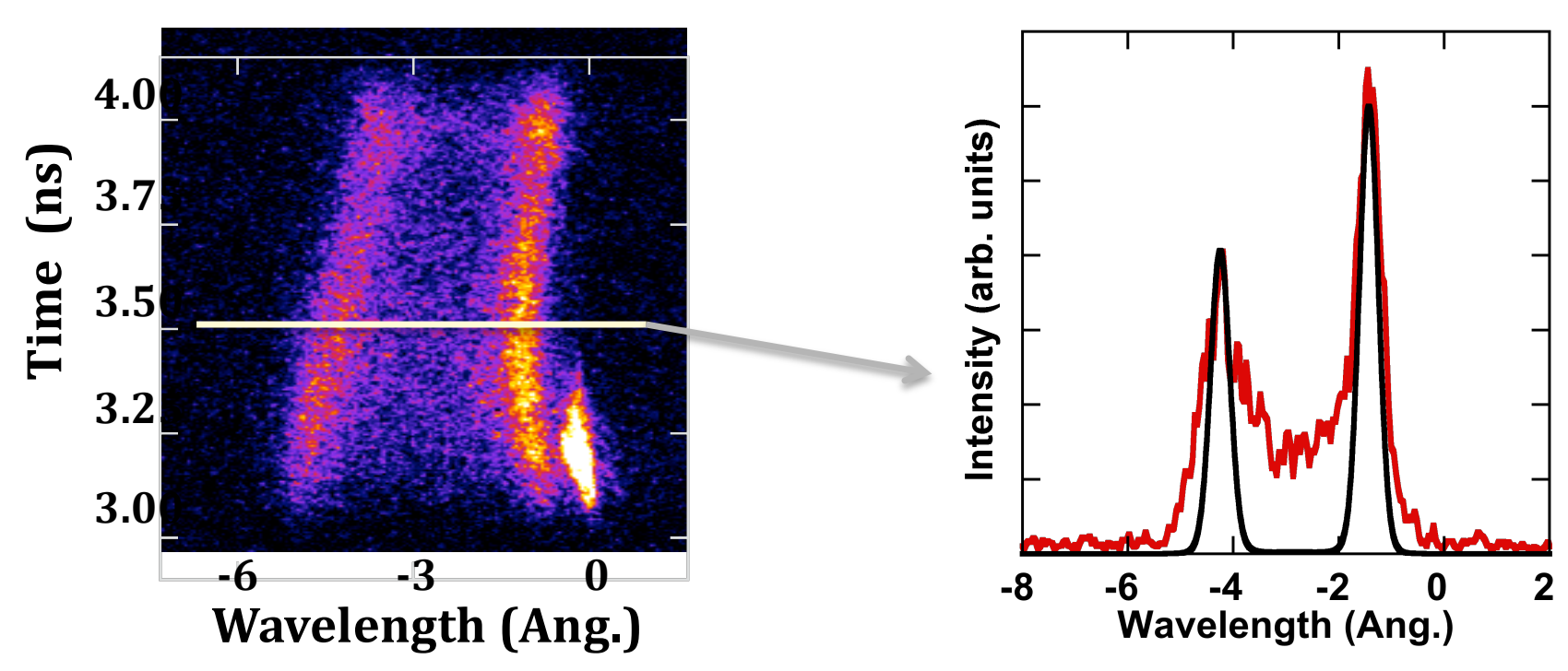

Figure XX: Ion feature data from $4^{\text {th }}$ harmonic Thomson Scattering, with fit by J.S. Ross for nickel plasma at $\mathrm{T}_{\mathrm{e}}=850 \mathrm{eV}$. 


\section{DD and $D^{3}$ He Yield Anomalies versus $D:^{3}$ He fuel ratio in Indirect-Drive Exploding Pushers (PI: Hans Rinderknecht, MIT) \\ with Chikang Li (MIT) and Peter Amendt (LLNL)}

Anomalous nuclear yield reduction as a function of $\mathrm{D}:{ }^{3} \mathrm{He}$ fuel ratio was observed in a study of $\mathrm{D}^{3} \mathrm{He}$-filled indirect-drive implosions performed on May $7^{\text {th }}, 2013$ using the OMEGA laser. Previous experiments in directly-driven implosions of $\mathrm{CH}$ capsules demonstrated anomalous reduction of the compression yield in $\mathrm{D}^{3} \mathrm{He}$ mixtures, relative to implosions filled with hydroequivalent fills of $\mathrm{D}_{2} \cdot{ }^{1} \mathrm{~A}$ similar yield anomaly has also been observed in shock yield from directly-driven thin glass targets. ${ }^{2}$ This study was designed to investigate whether these yield trends are also observed in indirectly-driven $\mathrm{SiO}_{2}$ shells filled with $\mathrm{D}^{3} \mathrm{He}$ mixtures.

Targets were 600 micron diameter $\mathrm{SiO}_{2}$ shells with 24.4 micron thick walls, fielded inside $2 \mathrm{~mm}$ long vacuum hohlraums with $1.6 \mathrm{~mm}$ OD and 50\% LEH. These targets were filled with mixtures of $\mathrm{D}_{2}$ and ${ }^{3} \mathrm{He}$ with initial mass density of $4.6 \mathrm{mg} / \mathrm{cc}$, with deuterium ion fraction of $0.2,0.5,0.8$ and 1 . Resulting compression yields demonstrated a trend of reduction in the DD-neutron and $\mathrm{D}^{3} \mathrm{He}$-proton yield-over-clean (YOC) as the deuterium ion fraction was decreased from pure $\mathrm{D}_{2}$ fill (see Figure 1); simulations were performed by Peter Amendt. The observed trend is comparable with direct-drive studies between deuterium fraction of 0.5 and 1 ; however unlike previous studies, the observed yield continues to decrease relative to hydroequivalent- $\mathrm{D}_{2}$ as deuterium fraction drops below 0.5 . A similar trend is also observed in $\mathrm{D}^{3} \mathrm{He}$-proton yields for targets with initial gas fill between deuterium fraction of 0.2 and 0.8 (see Figure 2). Implosions of targets with 50:50 mixtures of $\mathrm{D}^{3} \mathrm{He}$ and mass density of 2.9 and $1.5 \mathrm{mg} / \mathrm{cc}$ demonstrated a reduction in the observed DD-neutron YOC as pressure was reduced. These results confirm the anomalous reduction of compression yield in indirect-drive implosions of $\mathrm{D}^{3} \mathrm{He}$ fuel mixtures, supporting the hypothesis that multiple-ion plasma effects are significantly perturbing the evolution of indirect-drive implosions.

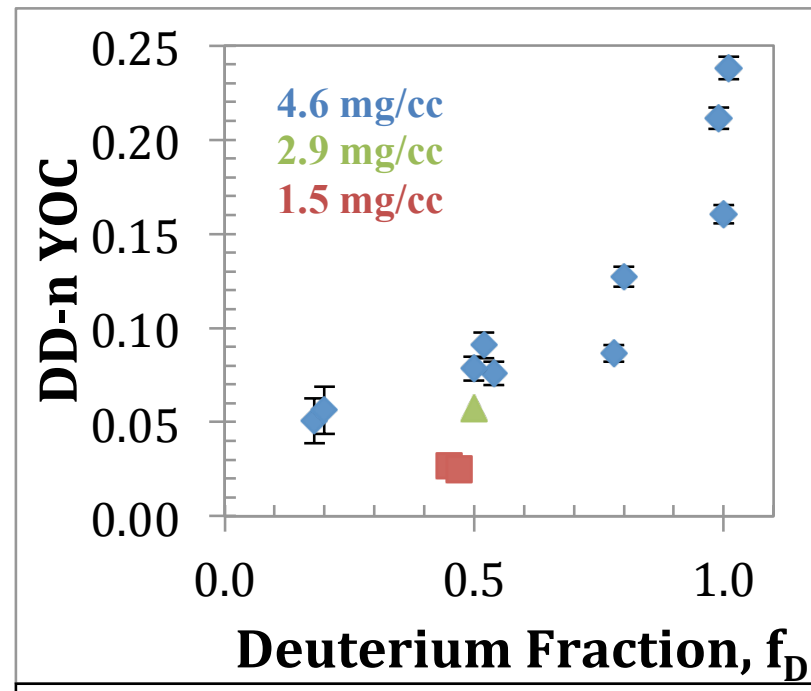

Figure 1: DD-neutron YOC versus deuterium fraction in indirect-drive hydroequivalent $\mathrm{D}^{3} \mathrm{He}$ fuel mixtures. Initial mass density is given for each set of implosions.

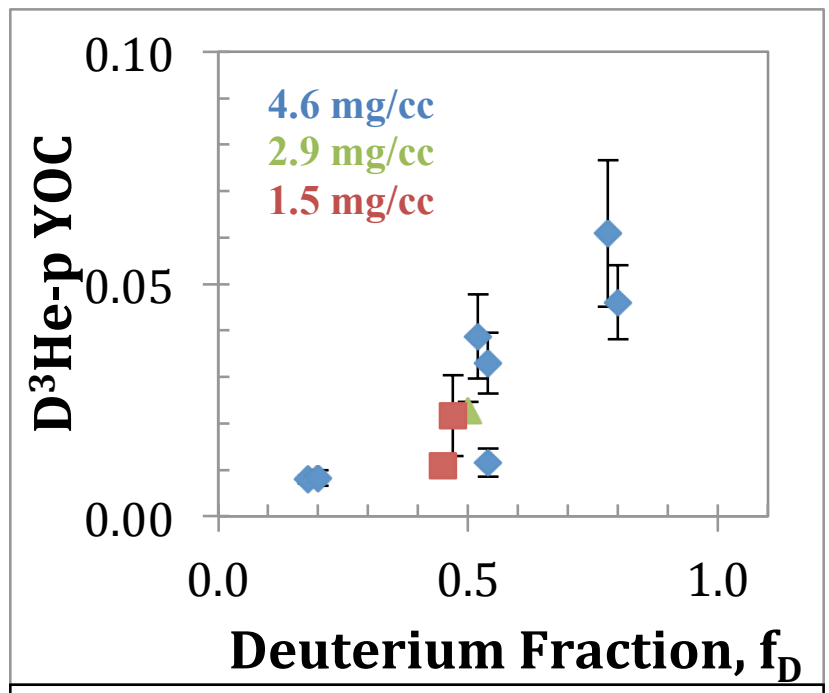

Figure 2: $\mathrm{D}^{3} \mathrm{He}$-proton YOC versus deuterium fraction in indirect-drive hydroequivalent $\mathrm{D}^{3} \mathrm{He}$ fuel mixtures.

${ }^{2}$ H.G. Rinderknecht, et al, to be submitted 


\section{Platform development for measuring charged-particle stopping in warm dense plasmas (PI: A. Zylstra, MIT) \\ with J.R. Rygg (LLNL)}

Charged-particle stopping power is a critical transport process for ignition and burn, and calculating DT$\alpha$ stopping in relevant plasma conditions depends on untested theories. To address the need to validate these theories, a platform is being developed on OMEGA EP to measure charged-particle energy loss. The experimental schematic is shown in Fig 1: the two short pulse beams are used to generate energetic protons via the TNSA mechanism, which heat and then probe a sample plasma. The FY13 experiments characterized the heating and probing protons separately via $\mathrm{x}$-ray radiography and charged-particle spectroscopy, shown in Fig 1. Future experiments will combine these techniques to measure the stopping power.

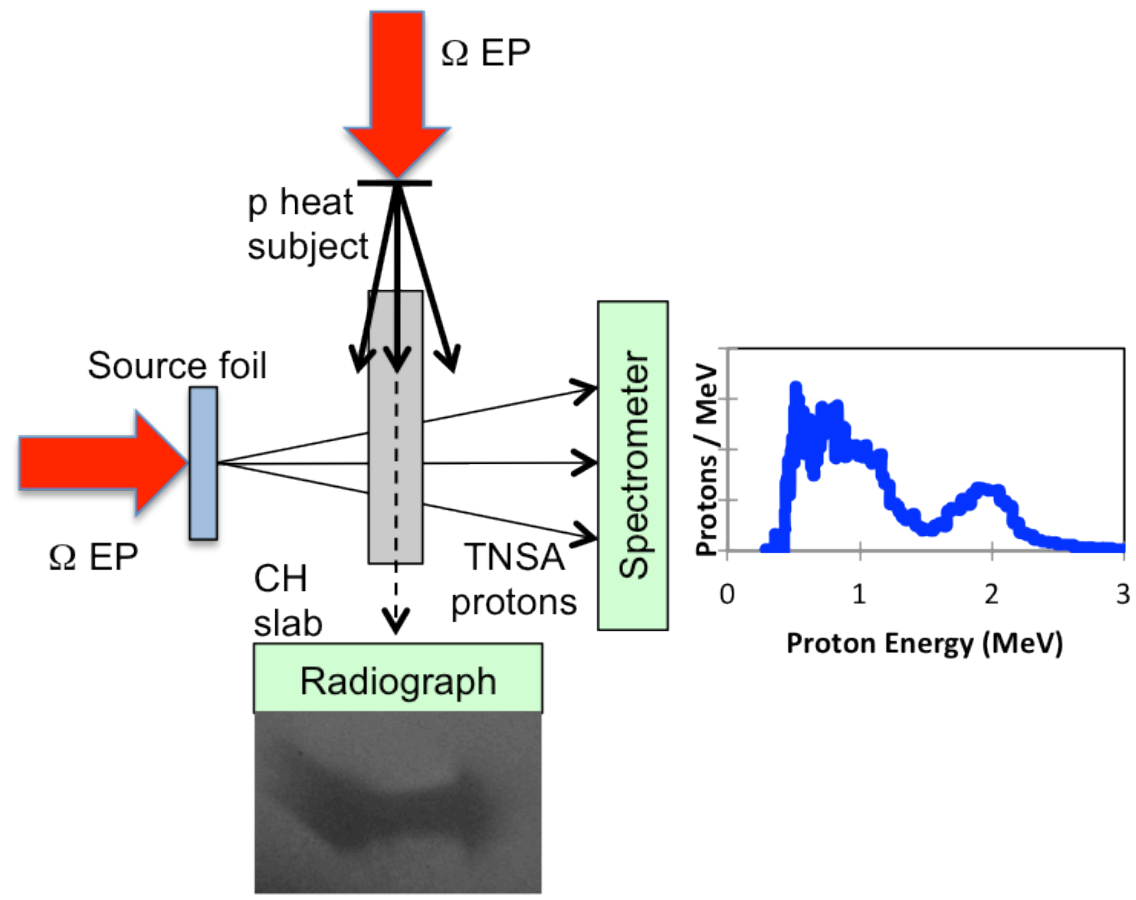

Fig. 1. Experimental schematic. One EP short pulse laser is used to heat a sample via proton isochoric heating, the second beam creates probing protons. The FY13 campaign characterized the isochoric heating with x-ray radiography (bottom) and studied proton source candidates for the stopping experiment (right). 


\section{High-Energy-Density Experiments}

\section{Material Dynamics and Strength}

\section{A. Tantalum Rayleigh-Taylor Experiments (PI: Chris Plechaty)}

The focus of the ICETaRT campaign was to study Ta material strength at high pressure ( $>1 \mathrm{Mbar}$ ), and high strain rates $\left(10^{6}-10^{8} \mathrm{~s}^{-1}\right)$. To achieve these conditions in an experiment, while keeping the temperature of the Ta sample well below melting, a reservoir and vacuum gap (Park 2010a) configuration was employed to produce a quasi-isentropic pressure drive (Figure 1). In the experiment, the plasma drive was produced by using 40 Omega beams to deposit $20 \mathrm{~kJ}$ of laser energy into a hohlraum. The x-rays produced in this process interact with the reservoir, to produce a plasma. The plasma then crosses the gap, and stagnates onto the physics package.

The physics package employed for these experiments consisted of a Ta sample, coated with a polyethylene $(\mathrm{CH})$ heat shield (to protect the Ta sample from the hot plasma). Imposed on the surface of the Ta were sinusoidal perturbations with $50 \mu \mathrm{m}$ wavelength and an amplitude of $1.7 \mu \mathrm{m}$ or $2.0 \mu \mathrm{m}$. In the experiment, as the plasma from the reservoir crosses the gap and stagnates on the sample package, the Ta ripples undergo an effective acceleration from the stagnating plasma drive. This acceleration actuates Rayleigh-Taylor (RT) growth of the pre-imposed sinusoidal pattern. Since the Ta material strength acts to suppress the amount of RT growth, measuring the RT ripple amplitude gives a measure of the material strength (Barnes 1974, Park 2010a, Park 2010b). To measure the perturbation amplitude, face-on high energy X-ray radiography (22 keV Ag k- $\alpha$ ) was employed using the EP short pulse beam. The EP beam was required in order to employ a high energy backlighter $(22 \mathrm{keV})$ to probe the high- $\mathrm{Z}$ tantalum material. To characterize the plasma drive, separate shots were taken using VISAR, where the physics package was replaced with a witness package.

The FY 2013 ICETaRT campaign (ICETaRT-13A) focused on studying the effect of crystal orientation on the material strength. In the multiscale model (Barton 2011), crystal orientation can affect material strength via a parameter known as the "Taylor factor". To understand the effect of crystal orientation on the material strength, we placed [100] and [111] oriented samples side-by-side and compared the ripple growth between these samples for the same shot, and under the same drive conditions. Initial results from these shots suggest that the difference in the growth factor between these two crystal orientations is too small to be measured within our error bars of $\sim 25 \%$. The implications of this are currently under investigation.

\section{References:}

Barton, N.R., et al., J. Appl. Phys., 109, 073501 (2011).

Park, H.S., et al., Phys. Rev. Lett., 104, 135504 (2010).

Park, H.S., et al., POP, 17, 056314 (2010).

Barnes, J. F., et al., J. Appl. Phys. 45 (2), 727, (1974). 


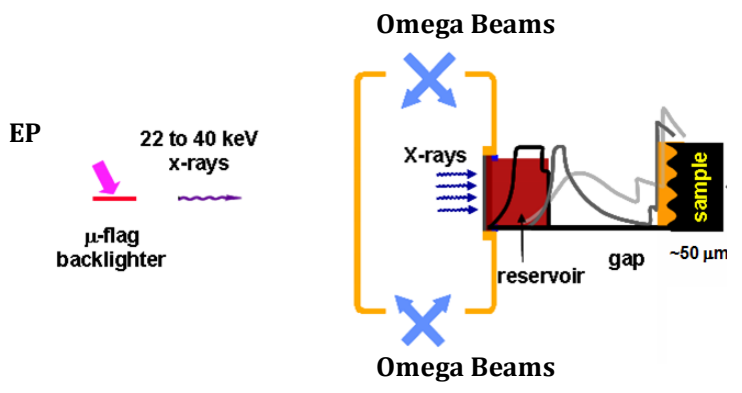

Figure 1. Experimental configuration employed for Omega-EP joint shots. Omega beams deposited energy into the hohlraum, producing a plasma drive. Face on high energy radiography was employed on the rippled Ta sample by using a short pulse EP beam to ablate an Ag backlighter. For Omega shots, the rippled Ta sample was replaced by a VISAR witness sample to characterize the plasma drive. 


\section{B. Iron Rayleigh-Taylor Experiments (PI: C. Huntington)}

The solid-solid phase transition between the bcc (alpha-) and hcp (epsilon-) lattice structures in iron is known to occur as the material is compressed. When kept below its melting point, an effective increase in the macroscopic strength of the material accompanies this phase transition. Understanding the material strength of iron throughout the deformation process presents a significant computational challenge, but is important for improving models of planetary structure, including interpretation of seismic measurements taken through our own Earth's core.

The IronRT OMEGA campaign used an indirect-drive platform to produce pressures of $\sim 1$ Mbar on a thin Fe disk with a sinusoidal ripple pattern machined on its face. These ripples seed the growth of the Rayleigh-Taylor (RT) instability, the magnitude of which is suppressed by the material strength of the sample. The amplitude of the ripples was diagnosed with high-energy X-ray radiography, using Ag micro-flags as the X-ray source. In addition to radiography, the VISAR diagnostic was essential to characterize the drive profile on the iron sample. On this IronRT shot day 5 radiography shots measured the Fe growth factors between 1 and 2. Comparison of these measured iron growth factors to a range of models for material strength in iron is ongoing. Importantly, the ability to reach $~ 1$ Mbar on OMEGA extends the accessible parameter space for this work, when combined with previous experiments conducted at $0.1-0.3$ Mbar pressures (J. Belof et al., AIP Conf. Proc. 1426, 1521, 2012).

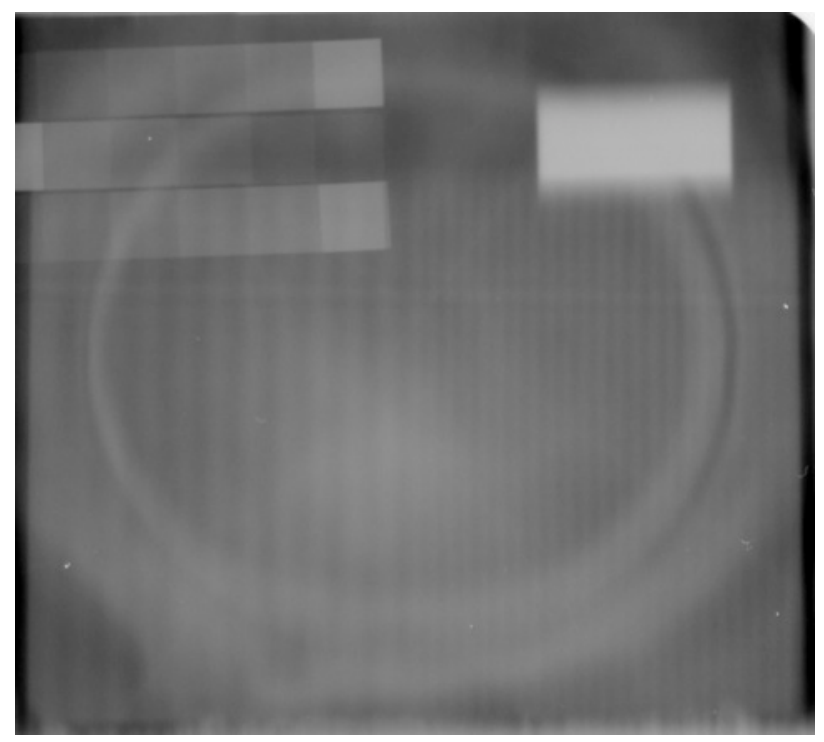

Figure XX: Example radiography data from the IronRT-13A campaign. The step features in the upper left allow for calibration of the detected X-ray signal with the material areal density $(\rho R)$ traversed by the X-rays. The opaque region in the upper right is a gold knife-edge for calculation of the modulation transfer function (MTF), with the remainder of the sample dedicated to the ripple measurement at two wavelengths (side-by-side 100 and 50 micron wavelengths). 


\section{Long-Pulse Silver Backlighter Development (PI: C. Huntington)}

The LPAgBL campaign was a half-day effort to quantify the spatial resolution achievable from a range of micro-flag x-ray source (backlighter) designs. Crucial for experiments in the Material Strength campaigns on NIF and OMEGA, the small (300 x 300 micron) silver foils produce a quasi-1D X-ray source when heated by the laser and viewed edge on, allowing for radiography of ripple growth in the strength experiments. On this shot day, each of 5 designs were irradiated with 18 beams, producing a total on-target intensity of $8 \times 10^{16} \mathrm{~W} / \mathrm{cm}^{2}$ and generating $\mathrm{Ag} \mathrm{K} \mathrm{K}_{\alpha}$ X-rays (near $\sim 22 \mathrm{keV}$ photon energy). From knife-edge radiography, the modulation transfer function (MTF) of each backlighter was measured. From this work it was determined that the MTF at a spatial frequency of 100 microns from a 3 micron-thick foil was twice that from a 10 micron-thick foil. The results of this experiment have been incorporated into several recent NIF target designs exploring the material strength of Ta. 


\section{Material Recovery Experiment (PI: M. May)}

The FY13 Material Recovery shots tested a prototype target recovery system that enables post-shot target analysis of material samples. A new recovery tube platform was qualified for use on OMEGA for these experiments. Five hohlraum target shots were performed. OMEGA was used to deliver $20 \mathrm{~kJ}$ of energy with 40 beams in a $1 \mathrm{~ns}$ pulse to heat these targets. Four hohlraum targets were used to drive samples into the recovery tubes. Two of the samples were driven into recovery tubes at lower pressure ( 1 MBar) and two were driven at higher pressure ( 2 MBar). One drive characterization target was shot to measure the high pressure drive using the VISAR. Analysis of the VISAR indicated that this pressure was $\sim 1.8 \mathrm{MBar}$. Platinum was used as driven sample material, since it is not common in laser produced plasma experiments and can be easily chemically extracted from the recovery tubes. To catch and hold the driven Pt, the recovery tubes were filled with two layers of increasing density (50 and $500 \mathrm{mg} / \mathrm{cc}$ ) dicyclopentadiene with a final layer of solid density $\mathrm{CH}$. The photographs show the pre-shot target mounted to the recovery tube, and the post-shot recovery tube. The postshot photo shows material residue on the recovery tube and a small gap between the target mount and the recovery tube. Post-shot analysis of the recovered samples indicated that $<10 \%$ of the Pt from the targets was collected in the recovery tubes. Valuable insight was gained regarding the participating physics and changes needed for future catcher designs involving a hohlraum.

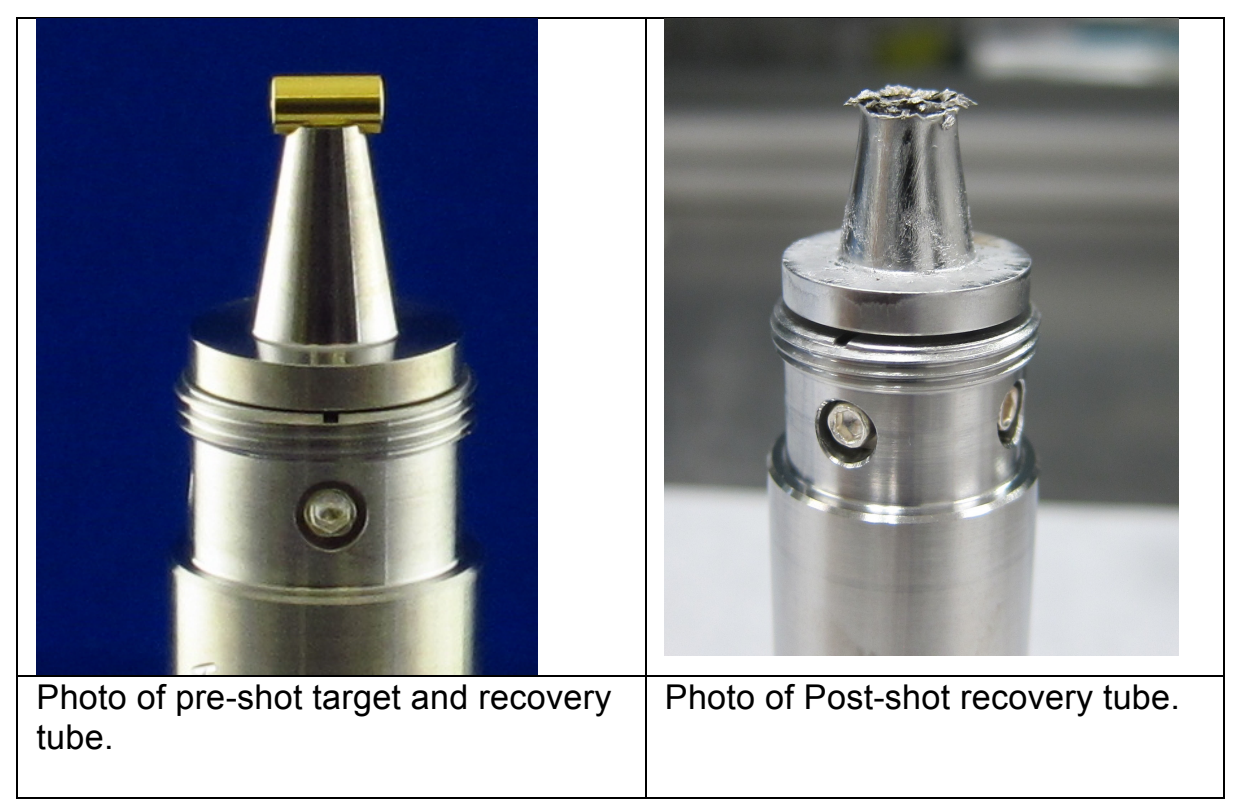




\section{E. Diffraction Studies on Shocked Tantalum (PI: C. Wehrenberg)}

Two distinct diffraction experiments were conducted to study the behavior of shocked tantalum.

The first experiment sought to make strength measurements of single crystal Ta samples which were shock compressed in the low pressure ( $<0.6 \mathrm{Mbar})$ regime. Previous campaigns (FY11, FY12) have explored up to $2 \mathrm{Mbar}$ shocks in Ta and successfully matched multi-scale model predictions; however, $\sim 0.6 \mathrm{Mbar}$ is expected to be a threshold for homogeneous nucleation of dislocations, which should alter the material behavior. In two half-day campaigns at Omega, 12 shots were performed in which an imploding $\mathrm{CH}$ capsule driven by 29 beams provided a broadband x-ray source. A single beam drove a thick $\mathrm{CH}$ ablator, with the shock wave attenuating as it propagated through the ablator to lower the shock pressure into the desired range. Time series of Laue patterns were recorded in the 0.2 to $0.8 \mathrm{Mbar}$ range. Figure 1 shows a series of Laue patterns for a 0.4 Mbar shock pressure. This series show the strain evolving from uniaxial compression to an ultimate flow stress. These lattice level measurements are directly comparable to multiscale model predictions.

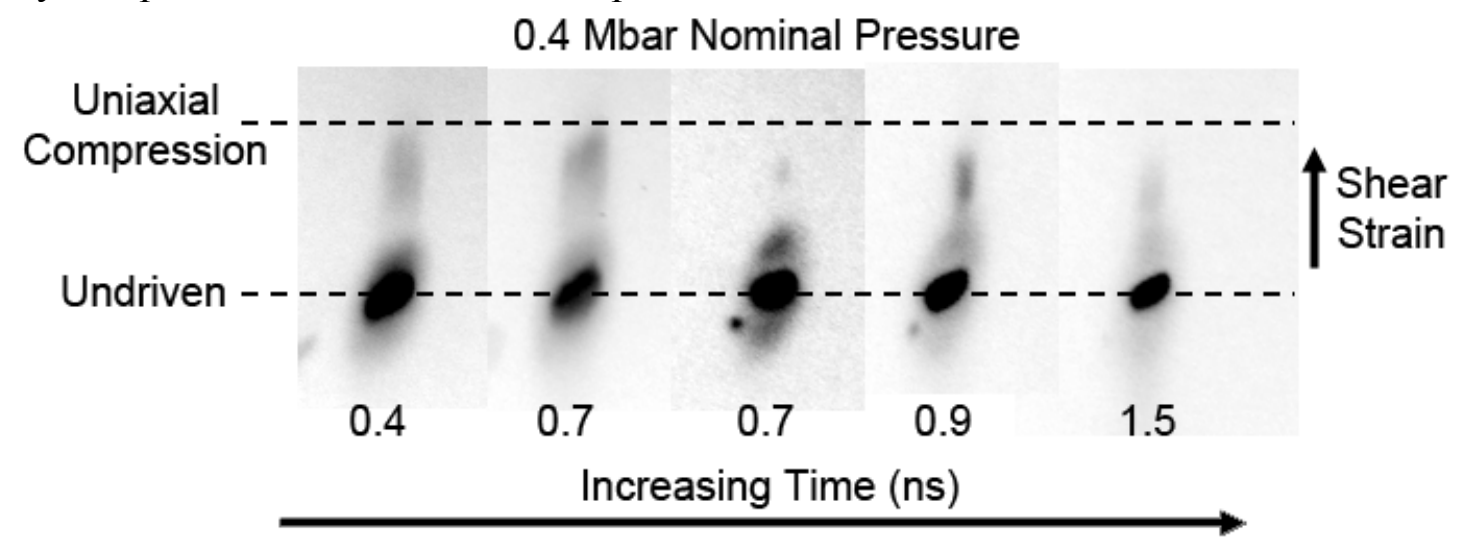

Figure XX. A time series of Laue images of single crystal Ta loaded along the (001) direction by a 0.4 Mbar shock wave. Shear strain is indicated by a shift in the diffraction spot from the undriven position. The time series shows the strain relaxing with time.

In parallel, a second class of experiment developed methods for recording diffraction patterns from polycrystalline samples using high-energy backlighters. In one shot day, a single short pulse (100 ps) beam drove the Zr backlighter foil. In a second shot day, 3 UV beams were used to drive a $\mathrm{Zr}$ foil backlighter with a 2 ns pulse. A separate UV beam was used to drive a target consisting of an HDC ablator, a Ta foil sample, and a high-density-Carbon (HDC) window attached to a heavymet pinhole mounted on the BBXRD (BroadBand X-ray Diffraction) diagnostic. No diffraction lines were recorded with the short pulse beam, due to a very high background. The long pulse shot day recorded up to 7 diffraction lines from the Ta sample, using backlighter laser intensities of $3-9 \times 10^{15} \mathrm{~W} / \mathrm{cm}^{2}$. Figure 2 shows a static shot (no Ta drive beam) using a $1 \mathrm{~mm}$ pinhole. Additional shots used a $0.5 \mathrm{~mm}$ pinhole to achieve greater angular resolution, but with a lower signal-to-noise ratio. These shots represent the first high-quality diffraction data recorded using high-energy, laser-driven x-ray sources. Driven diffraction data was recorded at $\sim 2$ Mbar; however, due to difficulties in timing the arrival of the shock wave, the diffraction was taken while the Ta was in the process of releasing to ambient pressure. Despite essentially splitting the signal between two strain states, two driven diffraction lines and 4 ambient lines were recorded. 
Figure XX. Static diffraction image from a Ta foil sample using a $\mathrm{Zr}$ backlighter ( $2 \mathrm{~ns}$ pulse) and a $1 \mathrm{~mm}$ pinhole.

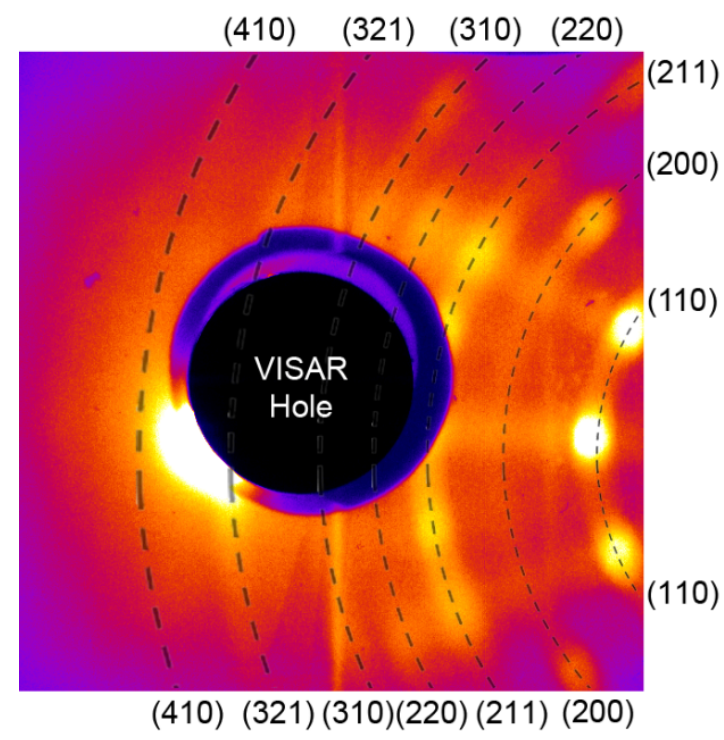




\section{F. Classical Rayleigh-Taylor Experiments (PI: H.-S. Park)}

The Classical Rayleigh-Taylor campaign is part of a continuing effort aimed at assessing the strength of various metals (primarily Ta and Fe) at high strain rates. Central to all of these experiments is a planar sample with a pre-imposed sinusoidal ripple on one surface. Under load generated by the laser drive, the rippled surface is unstable to Rayleigh-Taylor (RT) instability, and will grow in time to a perturbation that can be measured using face-on radiography. In the metal samples, strength of the material resists deformation and suppresses RT. However, to properly characterize the degree of resistance in metals, the ClassicalRT platform was developed to measure purely plastic RT evolution (that is, instability growth in the absence of material strength). On this shot day the rippled sample was made of brominated plastic $(\mathrm{BrCH})$, which is able to deform without any resistance from an underlying lattice structure. The VISAR and face-on X-ray radiography were the primary diagnostics, and the laser drive was generated via direct irradiation of a plastic ablator. Good data was collected using both primary diagnostics, and growth factors of approximately 10 have been calculated for the plastic sample. This experiment provided important "no-strength" data for the Materials Strength effort, and the remainder of the plastic dispersion curve will be measured in an upcoming FY14 shot day.
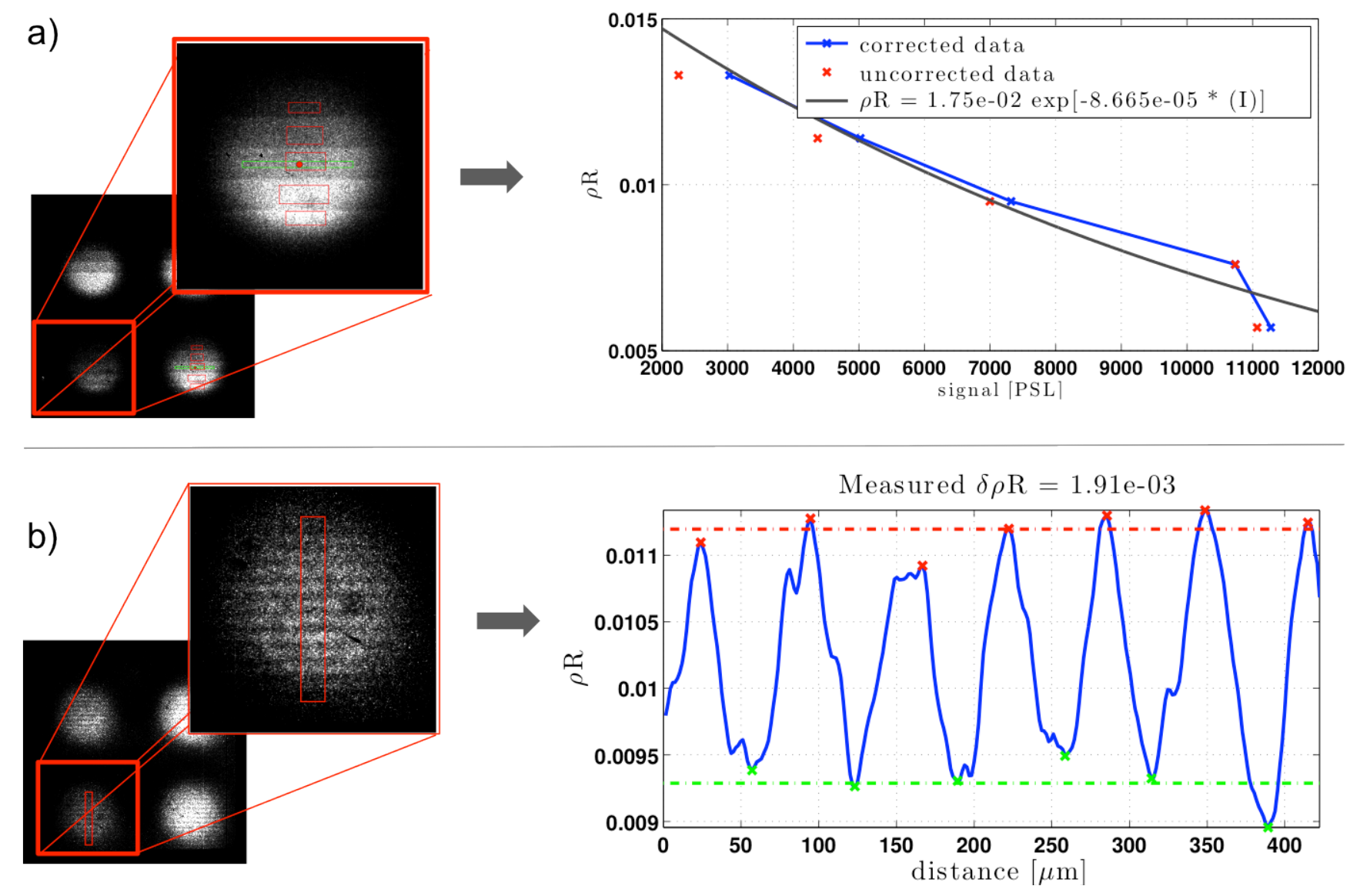


\section{Material Equation of State}

\section{A. Demonstration of single-shot EXAFS measurements on ramp compressed Ta (PI: Y. Ping)} with F. Coppari, B. Yaakobi, J. H. Eggert and G. W. Collins

Experiments in FY13 extended a recently published extended X-ray absorption fine structure (EXAFS) technique for dynamically compressed materials. The prior work on EXAFS measurements of dynamically compressed iron up to 560GPa (Ping et al. Phys. Rev. Lett. 111, 065501, 2013) has been extended to study ramp compressed Ta. EXAFS at the Ta L-edge is challenging because the signal is very small, on the order of $1 \%$ modulation in intensity. We have improved the backlighter brightness by $2 \mathrm{x}$ and the spectrometer efficiency by $5 \mathrm{x}$, so that one shot now is equivalent to 10 shots before. A specially designed quintuple-crystal spectrometer [Fig. 1(a)] acquires 5 spectra in a single shot [Fig. 1(b)], which can be averaged to improve the signal-noise ratio. EXAFS of both undriven and rampcompressed Ta at 1-3Mbar has been observed in single-shot measurements. A typical EXAFS spectrum is shown in Fig. 1(c). The data indicate that Ta undergoes a phase transition above 1Mbar. A paper summarizing these results has been submitted to Rev. Sci. Instrum. (Y. Ping, D. G. Hicks, B. Yaakobi, F. Coppari, J. Eggert and G. W. Collins, "A platform for XAFS study of dynamically compressed materials above 1Mbar".)
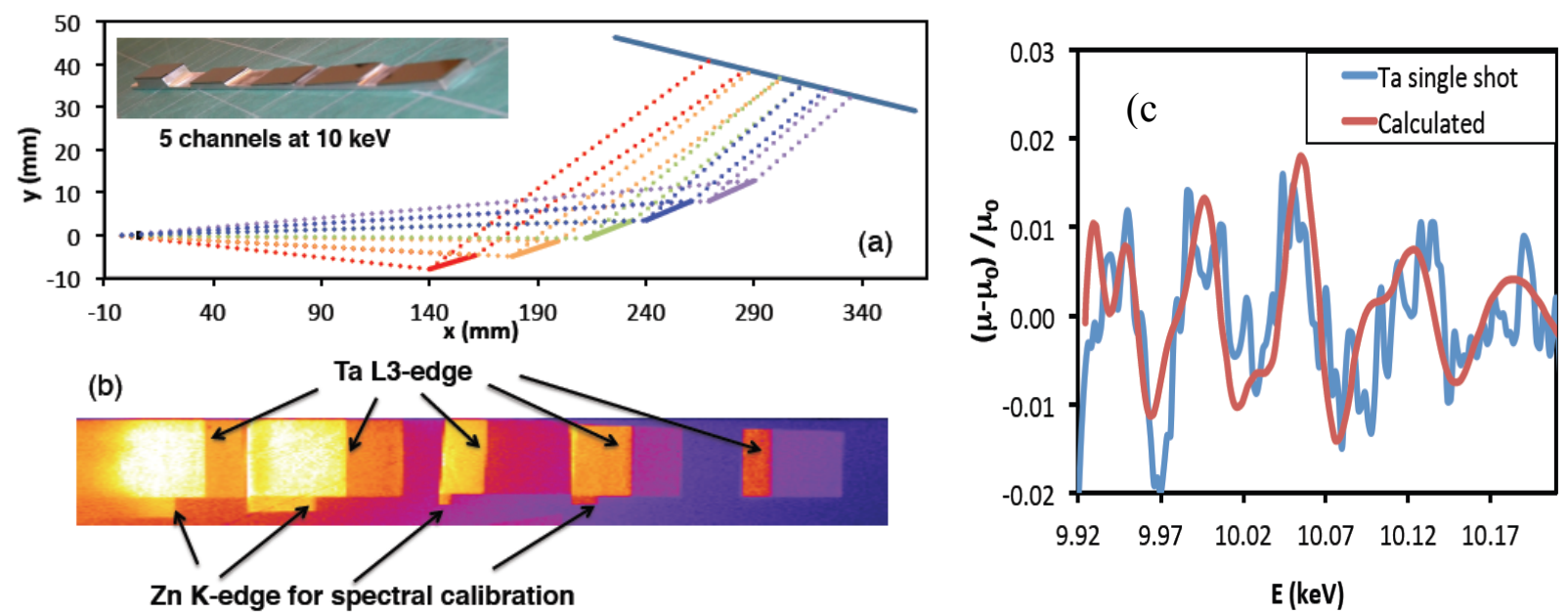

Figure XX: (a) Design of the quintuple-crystal spectrometer to record 5 spectra in one shot. The ray tracing results of the 5 channels are represented by different colors. A photo of the actual crystal is displayed at the up-left corner. (b) A raw image of the detected spectra, showing the 5 channels with Ta L3-edges and Zn K-edges as a spectral fiducial. (c) EXAFS data at Ta L3-edge taken by the quintuplecrystal spectrometer in a single shot and averaged over the 5 channels. 


\section{B. Tin Melting and Recrystallization (PI: A. Lazicki)}

The TinMelt campaign is an effort to detect and map out a recrystallization transition by shock melting a sample and then ramp-compressing it across the solid-liquid phase boundary. The metrics for recrystallization are (1) features in the profile of the ramped velocity history, measured using VISAR, that could indicate a phase transition, and (2) the presence of a solid x-ray diffraction signature after the recrystallization. Two half-day campaigns in FY13 searched for evidence of this transition in tin and also in lead. Lead is isovalent with tin, has a similarly low melting temperature, and is a much stronger $\mathrm{x}$-ray scatterer. These shots also experimented with new methods for optimizing the signal-to-noise on the image plates in our diffraction diagnostic. Efforts were made to increase the X-ray flux from the backlighter foil by (1) ablating it from both sides, (2) increasing the laser-to-X-ray conversion efficiency by adding a prepulse to the laser drive, and (3) increasing the laser-to-X-ray conversion efficiency by using a new converging cylinder geometry.

The targets for these shots were composed of tin or lead foils placed next to foils of high meltingtemperature tantalum. The two regions were subjected to the same shock-ramp pulse shape, to determine if the wave profile features could be associated with a solid-liquid transition (expected in the $\mathrm{Sn} / \mathrm{Pb}$ but not in $\mathrm{Ta}$ ). Diffraction from the shock-melted, ramped-compressed samples show a crystalline solid, indicating that recrystallization occurs over the timescale of the experiment. However, the wave profile results do not definitively show a unique feature that could be associated with this transition.

Vohra, Phy. Rev. B 42, 8651 (1990)

Mao, Solid State Comm. 74, 1027 (1990)

Figure 7. X-ray diffraction results from lead ramp-compressed from a shockmelted state

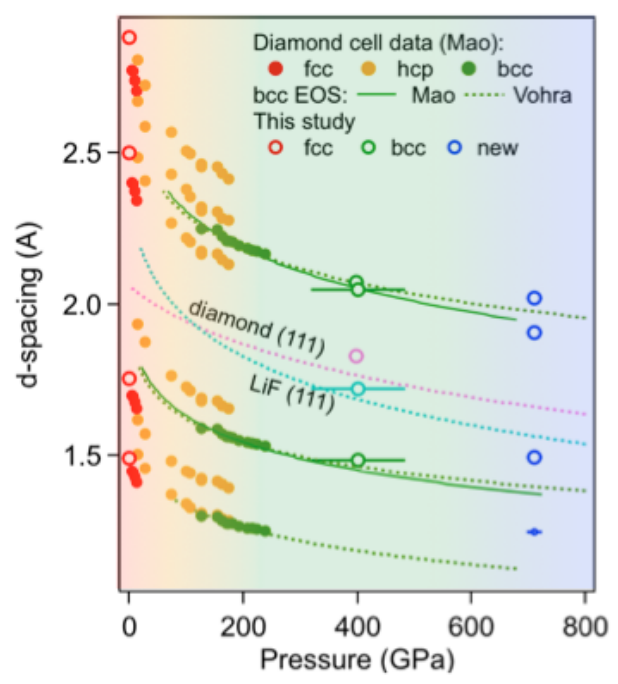




\section{Ta X-Ray Diffraction with Ramp Compression (PI: A. Lazicki)}

The TaDiff-13A/13B campaigns were designed to study a high pressure phase transition and equation of state in Tantalum and to improve methods for performing the diffraction measurements above 10 Mbars. During these campaigns, targets were ramp-compressed to pressures as high as 14 Mbars and Xray diffraction measurements were made using the PXRDIP diagnostic with a copper foil X-ray source. Pressure was determined from VISAR measurements. The 13 successful shots consisted of 8 tantalum targets, 2 tin targets, 2 lead targets and 1 iron target, all sandwiched between diamond plates, with indium or sputter-deposited gold heat shields. The tantalum shots were interspersed with tin, iron and low pressure lead shots to produce information needed to tune the ramp pulse shape and properly time the X-ray source. Diffraction data was obtained from tin between 11-13 Mbars, from lead at 7 Mbars, and from tantalum at 2 Mbars and 7-12 Mbars. Successfully tested were a new black kapton filter material, an x-ray preheat shield, and a new x-ray source configuration, all of which contributed to diffraction data that were much improved over previous campaigns. The data on $\mathrm{Pb}$ indicate a new high pressure phase transition, and the results on Sn further support a previously observed transition.
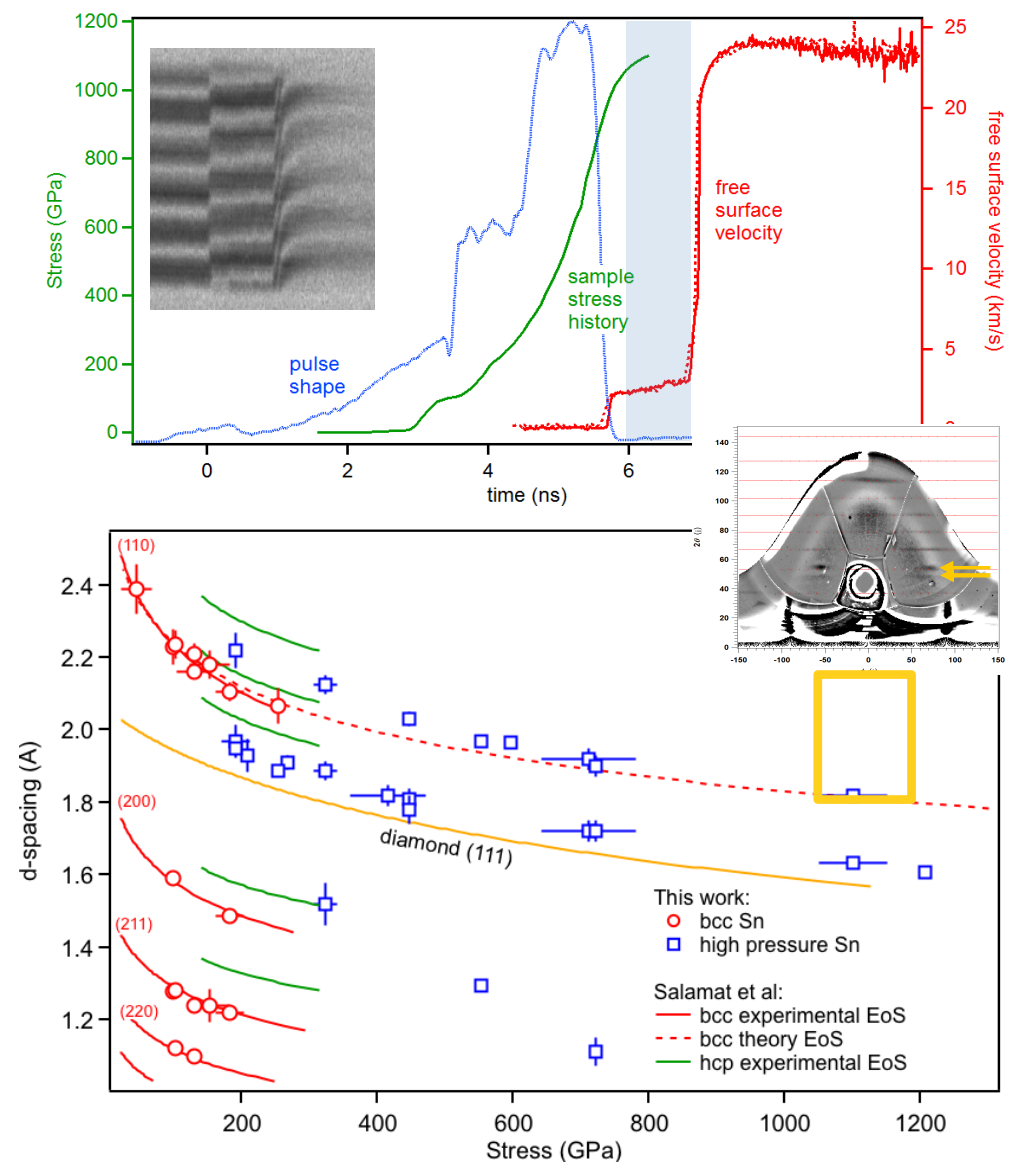

Figure XX: VISAR and diffraction data from tin above 10 Mbar, collected during the TaDiff-13B campaign 


\section{Development of a Soller Slit Diagnostic for Dynamic Diffraction Studies (PI: J. Hawreliak)}

The bulk of high-pressure research over the past century has been conducted in a diamond anvil cell (DAC) where pressure is applied by two opposing anvils. This technique is ultimately limited by the strength of the anvils, and for the strongest material, diamond, achieving and sustaining pressures above $300 \mathrm{GPa}$ is extremely difficult. Studying material states at higher pressures requires the use of dynamic compression techniques, which are not limited by the ability of an anvil to sustain the pressure, but instead rely on inertial confinement of the material to sustain great pressures temporarily. Dynamic compression is ultimately limited by the energy density which can be imparted to the sample before it disassembles, or the pressure is released. In situ $\mathrm{X}$-ray diffraction has become an important tool for probing the atomic structure of these materials at ultra-high pressure states. The most extreme environments associated with laser driven dynamic compression experiments require unique diagnostic capabilities, and in FY13 the ADVXRD campaign tested a Soller Slit diffraction diagnostic.

A conventional Soller slit on a synchrotron is a single pair of thin high-Z metal plates (typically tantalum) used to isolate a single line of sight. The direction of the slit is adjusted over a range of angles, and a measurement is made of a static sample. This reduces noise by allowing only signal that follows a particular path to fall on the detector. For laser-based experiments, one can use an array of Soller collimators, to provide a range of angles for a single experimental event. Figure 1a) shows the array of Soller collimators used over the detector, giving an effective pixel size of 400 microns. This reduces the background and allows diffraction to be recorded from a dynamically driven sample without collimating the beam at the target. There are two experimental advantages of this technique over collimating at the target. The first is that the target can be simplified, because it does not need to include a collimator. The second advantage is reduced drive noise, because the collimation happens farther from the X-ray sources, i.e. not at the sample, but at the detector. Figure 1b) shows the diffracted signal from an undriven tantalum sample. The alignment was such that some pixels had direct line of sight of the backlighter and are saturated. But the diffraction ring is visible. This data will be used to benchmark the diagnostic and drive further development to improve the signal-to-noise and angular resolution. Future experiments are envisioned to use this Soller Slit collimator with a hohlraum driven sample.

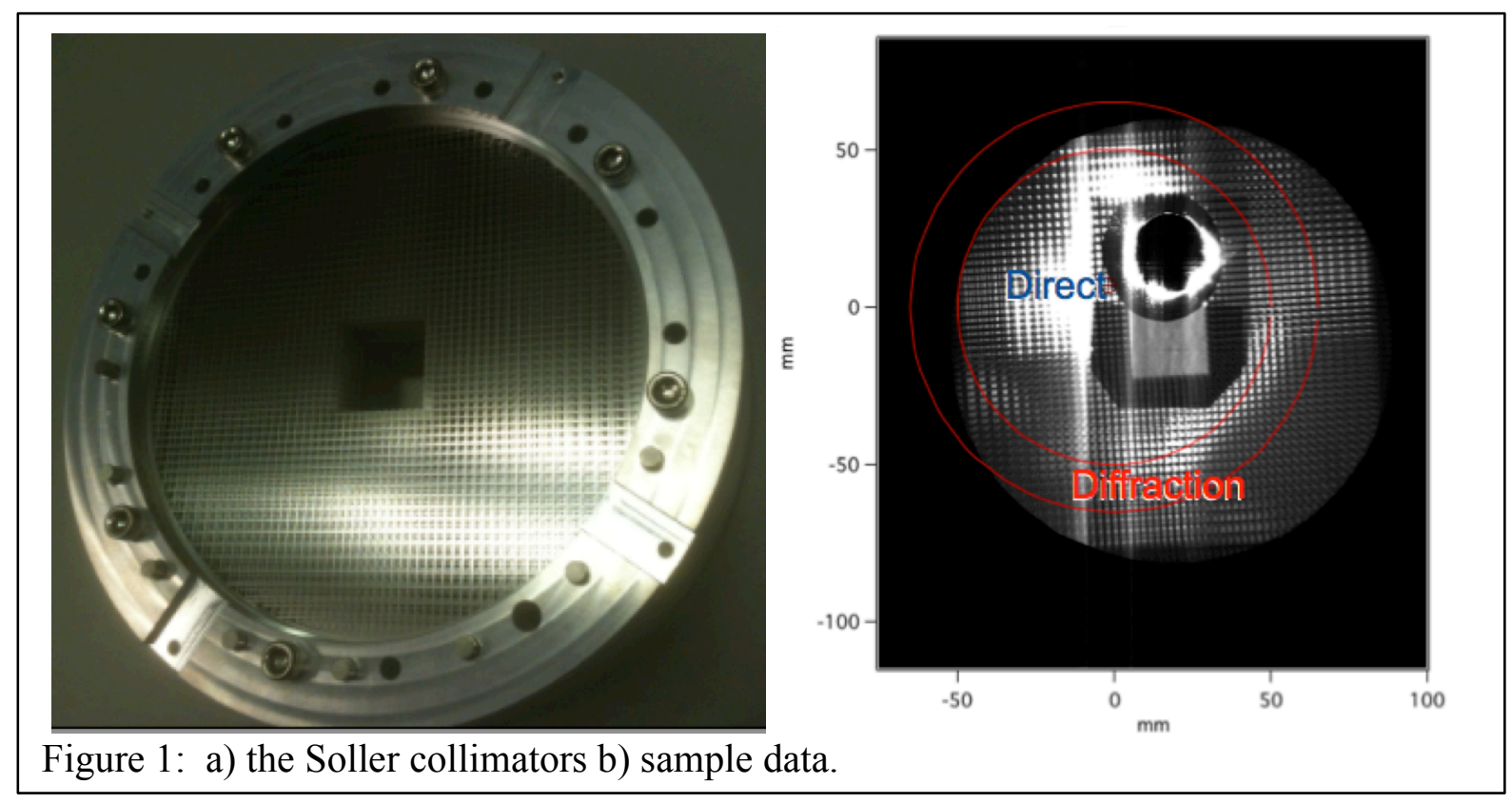




\section{E. Radiographic Equation of State Measurements of Shocked Foams (PI: J. Hawreliak)}

Materials in extreme conditions generated by shock compression of aerogel foams are an important area of high energy density science. LLNL and its partners have developed the capability to engineer low density foams with the desired properties for a range of programmatic and scientific experiments. The initial density of the foam is used to set the thermodynamic path of the shock Hugoniot. For laboratory astrophysics and other HED experiments, where low density aerogels are used to model interstellar matter, a detailed understanding of the EOS is important. However, continuum EOS theories make assumptions about bulk behavior of these foams which ignores the underlying fact that foams are fundamentally a heterogeneous material at small length scales. Continuum EOS models can not simply be scaled by density scaling, thus requiring foam EOS Hugoniot measurements. Furthermore, many low-density carbon and $\mathrm{CH}$ based foams of interest are not transparent, making impedance-matching experiments difficult and less accurate. To avoid these issues, a platform has been developed for in-situ radiography of shocked foams, from which the shock speed and density can be measured.

Figure 1a) shows a VISRAD model of the experimental configuration. Two or three beams are used to generate a K-shell area backlighter from a material chosen to deliver sufficient photon flux to the detector with an X-ray energy low enough for the foam sample's absorption cross section to yield good contrast at the shock front. For $50 \mathrm{mg} / \mathrm{cc} \mathrm{CRF}$, the $\mathrm{Cl} \mathrm{He}-\alpha \mathrm{X}$-rays from Saran are used as the backlighter. A second set of beams aimed onto a quartz drive plate generates an expanding spherical shock front. The spherical shock front removes parallax issues associated with the different viewing directions of different strips on the framing camera. Figure 1b) shows a single frame from the $\mathrm{x}$-ray framing camera identifying the shock front and the opaque quartz pusher.
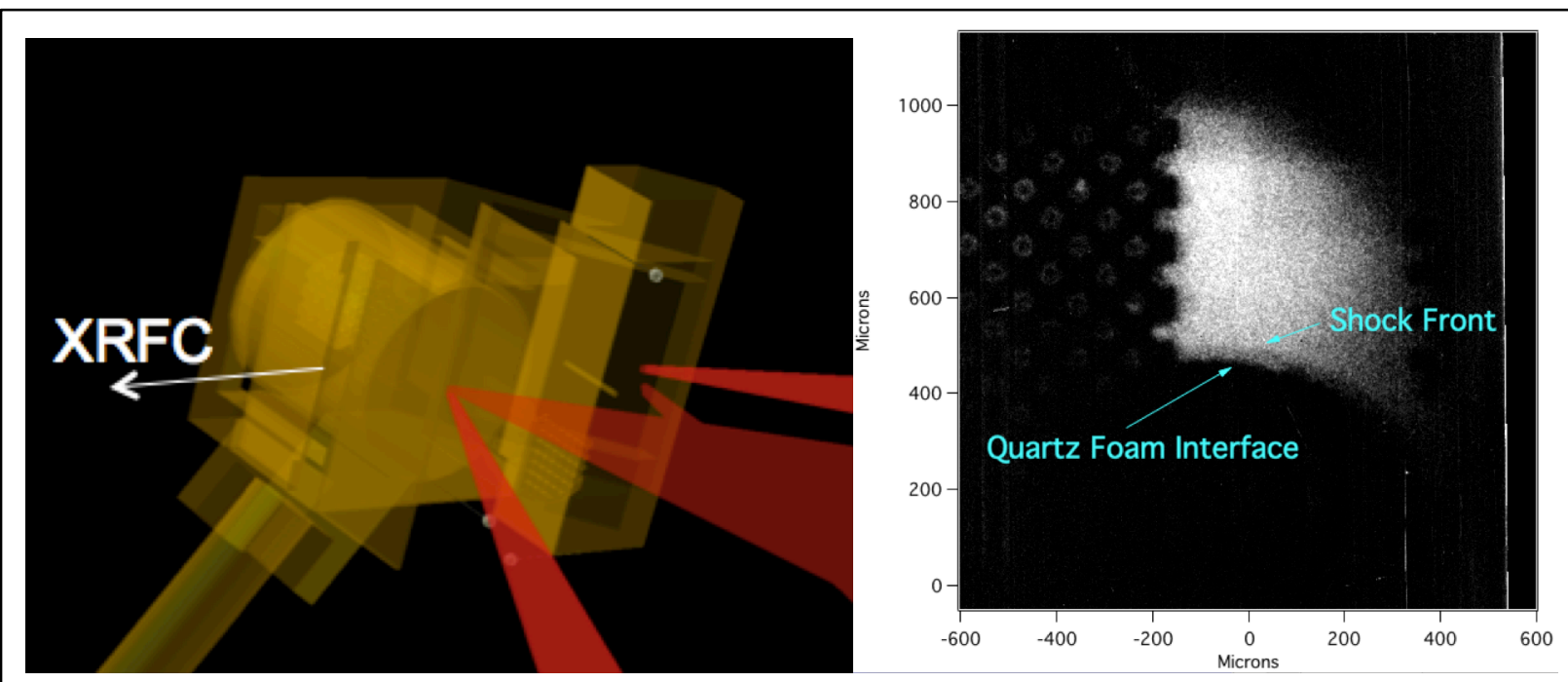

Figure 1: a) VISRAD model of experiment. B) Single frame from 2-strip framing camera 


\section{F. High Energy X-ray Diffraction Development (PI: J. Hawreliak)}

In situ X-ray diffraction is a powerful diagnostic technique for probing atomic structure at high pressures. Cutting edge molecular dynamic simulations show that structure becomes more complex at higher pressures, requiring shorter-wavelength X-ray sources to uniquely determine the phase. K-shell emission from short pulse lasers such as Omega-EP is a technique for generating high energy $\mathrm{X}$-rays, but also produces a high energy bremsstrahlung spectrum that generates background. Although techniques have been developed which use short pulse X-ray backlighters for radiography, the use of similar backlighters for X-ray line diffraction, in the HighEXdiff campaign, was not successful. To identify the failure mode, one set of Ross-pair filters was used to isolate tungsten L-shell fluorescence, while a second pair isolated $>50 \mathrm{keV}$ X-rays. The data showed that the predominant source of strong background is from X-rays with energies $>50 \mathrm{keV}$. Tungsten was the primary material used for $\mathrm{X}$-ray shielding, and its $\mathrm{K}$-shell fluorescence at $59 \mathrm{keV}$ appears to be the dominant source of background in the BBXRD diffraction diagnostic, rather than direct $>50 \mathrm{keV}$ X-rays from the backlighter. Figure 1 shows the Ross pair images on the image plate.

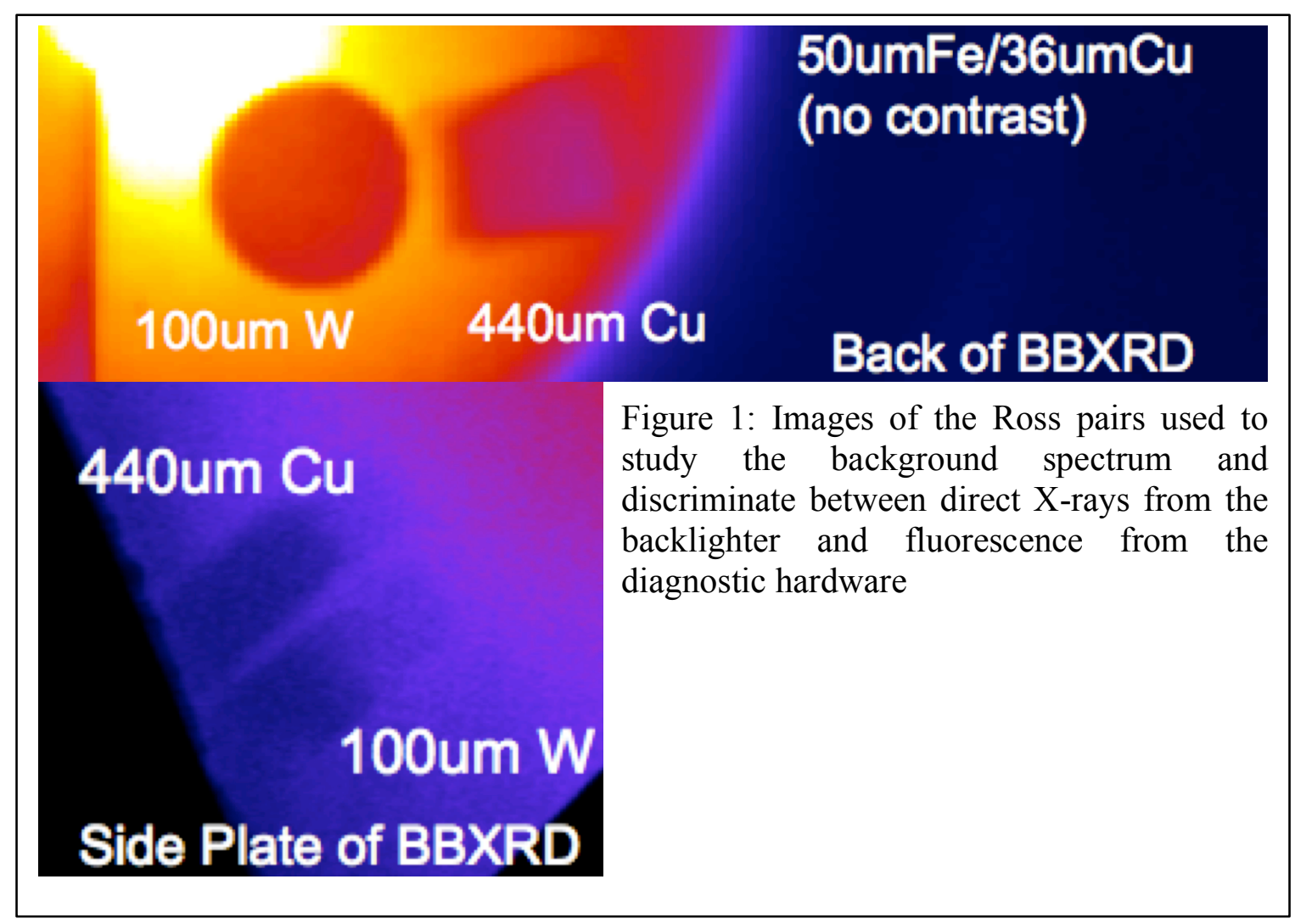




\section{G. Lithium Hydride Equation of State (PI A. Lazicki)}

The LiHEOS campaigns represent an effort to measure the shock Hugoniot of LiH, for the purpose of constraining theoretical models. Two methods were pursued in FY13. The first used the VISAR velocity diagnostic to track reflecting shock waves in targets composed of single crystal quartz and $\mathrm{LiH}$, relying upon impedance-matching to the quartz standard to determine the Hugoniot data points. The other method uses radiographic imaging to capture the motion of the shock front in LiH using a framing camera. Several radiography geometries were investigated: imaging through pinholes or slits with an area backlighter, and a point projection configuration.

The VISAR measurements were demonstrated to be successful, and highly valuable data was acquired. This was in spite of challenging issues with sample contamination (due to LiH's reactivity with moisture), and also with the VISAR technique (due to the large index of refraction mismatch between the two materials, which can create spurious reflections off of LiH-quartz interfaces).

Furthermore, after addressing debris, signal strength and target alignment issues, successful radiographic measurements were demonstrated, using an area backlighter and imaging the shock front through narrow slits.

References:

Ragan, LA-UR-83-2081.

Hama et al., PRB 39, 3351 (1989).

Kritcher et al., POP 16, 056308 (2009).

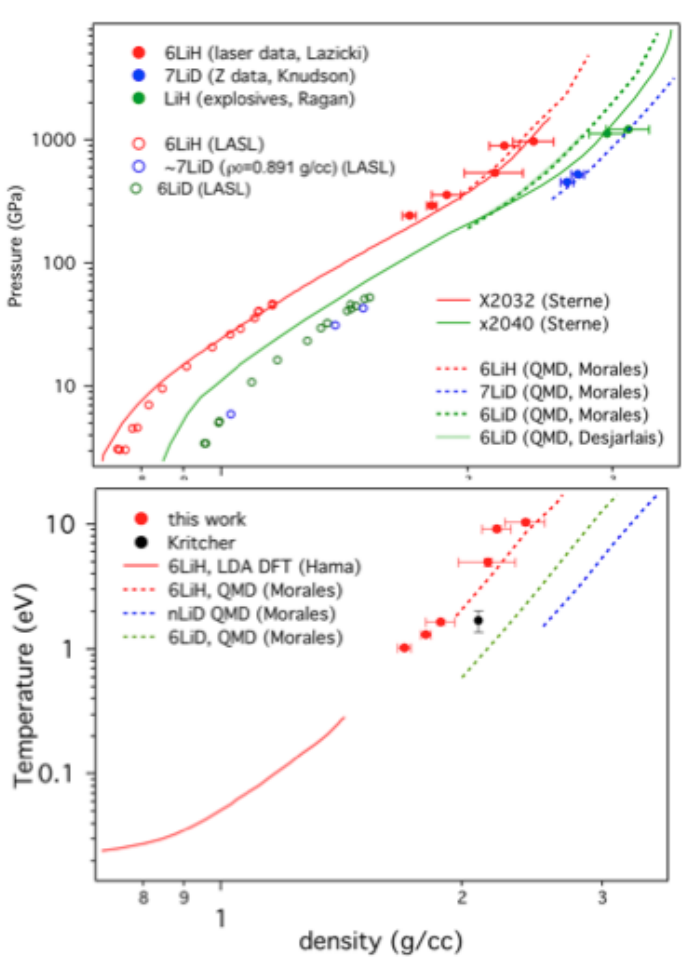

Figure 8. Pressure and temperature of $\mathrm{LiH}$ along the Hugoniot measured using VISAR and SOP

\section{H. Ta Equation of State (PI D. Fratanduono)}

Six experimental shots were conducted at the OMEGA laser facility to measure the shock Hugoniot of Ta. VISAR data was collected. 


\section{Radiation Transport and Opacity}

\section{A. Heated Wall Radiation Transport (PI: K. Baker)}

A campaign of six shots was carried out on the OMEGA laser to measure radiation transport in a geometry that minimizes wall loss. In these experiments a heat wave was propagated axially down a 30 $\mathrm{mg} / \mathrm{cm}^{3} \mathrm{SiO}_{2}$ foam, $700 \mathrm{um}$ in diameter by $1.7 \mathrm{~mm}$ in length, whose outer diameter was enclosed by a 70 um thick $\mathrm{Ta}_{2} \mathrm{O}_{5}$ annular foam. This foam structure was then placed inside a $1.6 \mathrm{~mm}$ by $2.9 \mathrm{~mm}$ long hohlraum and radiatively heated by the x-ray flux driven by laser interaction with the hohlraum walls. A supersonic heat wave was driven axially down the length of the $\mathrm{SiO}_{2}$ foam, and a transonic heat wave was driven radially inward through the $\mathrm{Ta}_{2} \mathrm{O}_{5}$ foam. The radially propagating transonic heat wave minimizes the wall loss for the axially propagating supersonic heat wave, enabling it to propagate over longer distances.

In these experiments a heat wave was successfully propagated over a distance greater than twice the Rosseland mean free path, and more than twice the diameter of the foam cylinder in which the heat wave propagated, as shown in Fig. 1a. A control target was also employed, which consisted of an inner $\mathrm{SiO}_{2}$ foam surrounded by 25 um thick gold annular walls to prevent a radially propagating heat wave from reaching the inner $\mathrm{SiO}_{2}$ foam. As seen in Fig. 1b, no heat wave was observed for this control target.
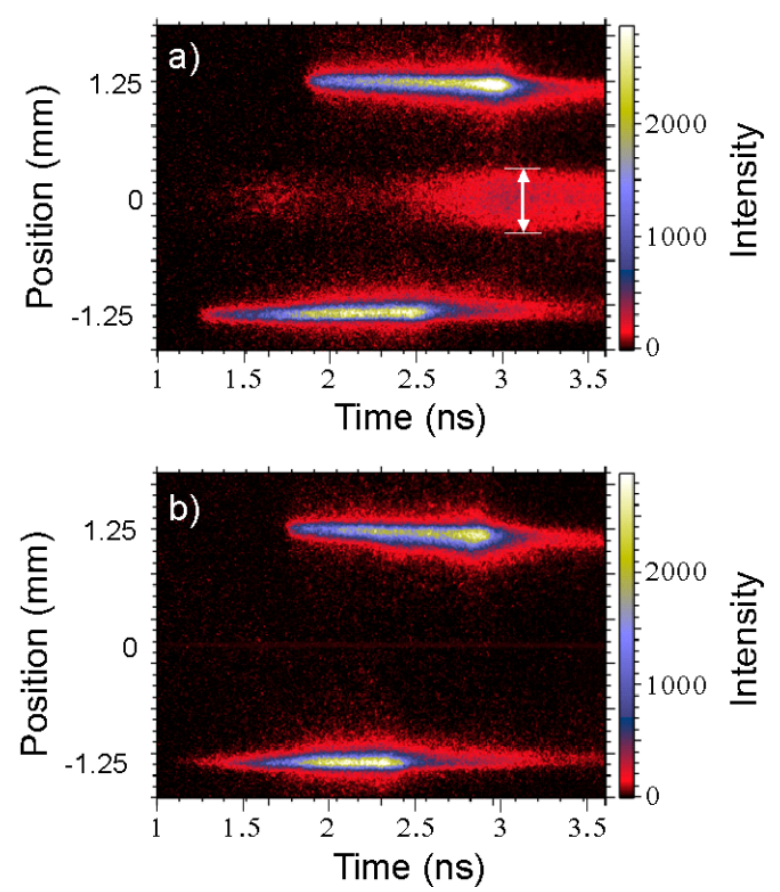

Figure 1 Heat wave signature measured at the end of the $\mathrm{SiO}_{2}$ foam for the case of $\mathrm{Ta}_{2} \mathrm{O}_{5}$ walls (a) and gold walls (b). The features at the $1.25 \mathrm{~mm}$ and $-1.25 \mathrm{~mm}$ positions represent the two timing lasers, which were incident at 1.75 and $1.25 \mathrm{~ns}$, respectively. 


\section{Hydrodynamics}

\section{A. Toto - Radiography Development for NIF Hydrodynamics Experiments (PI: V. Smalyuk)}

Backlighting experiments were performed on the OMEGA laser system to study symmetry of foam balls illuminated with hohlraum X-rays. Backlighter targets were driven with the Omega-EP short-pulse IR beam in Joint experiments. In the Joint configuration, 10- $\mu \mathrm{m}$ thick Ag wires, mounted on $300 \times 300 \mu \mathrm{m}$ square, $10-\mu \mathrm{m}$ thick polyimide foils were irradiated with $\sim 1.2 \mathrm{~kJ}$ short-pulse $(\sim 100 \mathrm{ps})$ IR beam at a laser intensity of $\sim 1 \times 10^{17} \mathrm{~W} / \mathrm{cm}^{2}$. X-ray radiographs of targets were measured on image plates using the HERIE diagnostic placed $\sim 50 \mathrm{~cm}$ from the sample, yielding a magnification of $\sim 50$. In addition, tests were performed using the short-pulse backlighting parameters that will be used on future National Ignition Facility (NIF) experiments backlit by the short-pulse ARC beam. This latter configuration adjusted the EP short-pulse beam to deliver $1.0 \mathrm{~kJ}$ of IR light with a 50ps pulse shape and an intensity of $\sim 2 \times 10^{17} \mathrm{~W} / \mathrm{cm}^{2}$. The radiograph signal and contrast with the ARC-like beam parameters were consistent with previous experiments, and showed increased resolution $(13 \mu \mathrm{m})$ compared to experiments with the nominal backlighter parameters ( $19 \mu \mathrm{m}$ resolution), as shown in Figure 1. These experiments were very successful; they produced high-quality radiographs that created a baseline for future Complex Hydrodynamics experiments on the NIF.

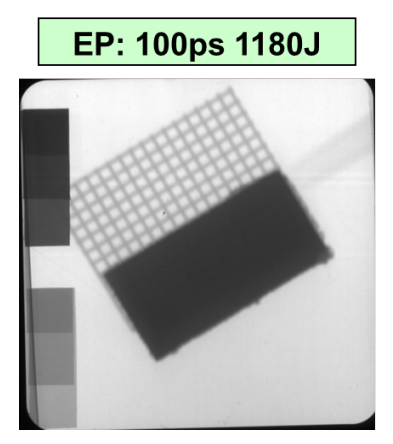

S70439 Resolution: 19um
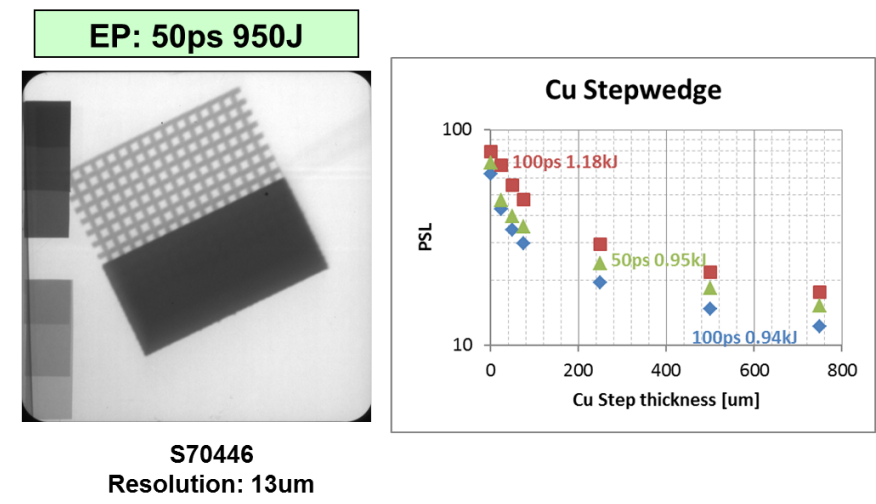

Figure 1: Backlighter performance data using a gold foil-grid radiography target and a $\mathrm{Cu}$ step wedge. 


\section{B. Copper Foam Shock Breakout Measurements (PI: A. Moore)}

High-Z metal foams made via a copper ceramic casting process, and with approx. $1 / 10^{\text {th }}$ solid density $(\sim 1 \mathrm{~g} / \mathrm{cc})$ have been developed at LLNL and are being produced routinely at AWE. These provide a novel target material for use in high-energy density physics experiments on NIF, Orion and OMEGA, but need to be well-characterized with a good understanding of the material opacity and equation of state (EoS) in order for experimental results to be constraining to radiation hydrodynamics simulation codes. With advances in the manufacturing techniques it has been possible to produce foam material with an average pore diameter of $1 \mu \mathrm{m}$ and less. In the five experiments conducted in the CuFmDrive-13A campaign described here, comparisons were made of the shock propagation in two materials, $1.0 \mu \mathrm{m}$ and $0.5 \mu \mathrm{m}$ pore foams, as a means to qualify the $0.5 \mu \mathrm{m}$ foam for use in future experiments.

The Rankine-Hugoniot relations for a single shock demonstrate the simple dependence of the shock velocity on the pressure achieved in the shocked foam, which is in turn dependent on the internal energy of the foam. Given this dependence, measuring the propagation of a single shock through a material sample has been developed as a reliable method to quantitatively validate the EoS model for a given material.

The platform to measure the shock transit-time in $\mathrm{Cu}$ foams was developed using a $5.5 \mathrm{~kJ}$ laser drive from 15 beams of the 60-beam, 30-kJ, 351-nm OMEGA Laser System. In these experiments the target package consists of a washer containing two foam samples, with a polystyrene ablator and aluminum flashing to improve the VISAR laser reflection. The ablator side faces the drive hohlraum The copper foam samples are $0.7 \mathrm{~mm}$ in diameter and nominally $0.25 \mathrm{~mm}$ in thickness with a nominal density of $0.9 \mathrm{~g} / \mathrm{cc}$. The hohlraum used is $1.6 \mathrm{~mm}$ in diameter and $1.0 \mathrm{~mm}$ long, with $1.2 \mathrm{~mm}$ diameter holes at each end (one for laser beam entry and one to drive the ablator), and reaches a peak temperature of $208 \pm 5 \mathrm{eV}$. Data using the streaked optical pyrometer (SOP) was obtained on five shots, an example of which is shown in Figure 1(b). The average shock velocity for the $1.0 \mu \mathrm{m} \mathrm{Cu}$ foam was $51.0 \pm 3.2 \mathrm{~km} / \mathrm{s}$ which is compared to $50.3 \pm 3.0 \mathrm{~km} / \mathrm{s}$ measured with the $0.5 \mu \mathrm{m}$ foam, indicating that a reduction in the pore size does not significantly alter the material EoS of the $\mathrm{Cu}$ foam.

In general, comparison of the results with post-shot simulations are favorable, again indicating that there are no significant differences in EoS. That said, limitations in the pre-shot characterization of the sample led to a large uncertainty in the sample thickness, allowing for a wide range of possible breakout times. A follow-on campaign in FY14 will extend the measurement to include shock velocity using VISAR, and will repeat some shots following better characterization of the sample thickness.

(a)

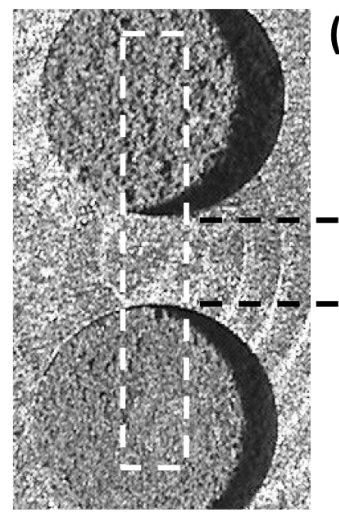

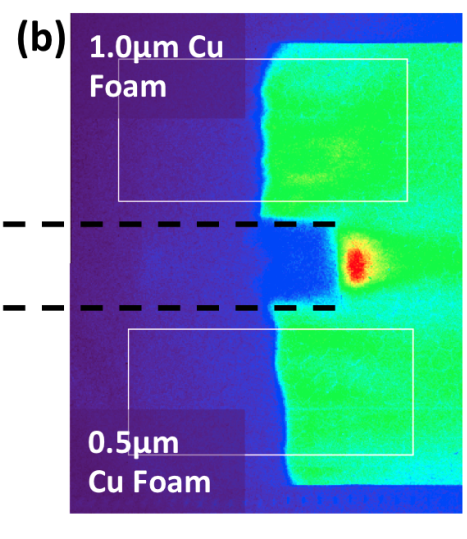

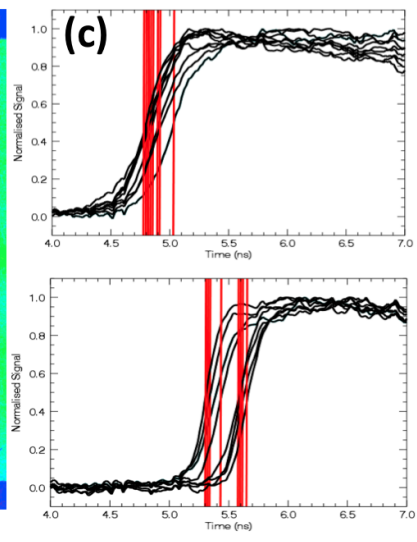




\section{Short-pulse, UV backlighting development for NIF (PI: D. Martinez)}

A series of eight shots were performed on OMEGA-EP in order to optimize, for NIF experiments, a point projection backlighter technique using a $\mathrm{Zn}$ backlighter foil, apertured with a $20 \mu \mathrm{m}$ pinhole tilted $30^{\circ}$ from the normal of a $50 \mu \mathrm{m}$ thick, $5 \mathrm{~mm}$ x $5 \mathrm{~mm}$ square Ta plate. The $\mathrm{Zn}$ foil was suspended from the Ta pinhole, with either $15^{\circ}$ or $37^{\circ}$ angle between the $\mathrm{Zn}$ foil normal and the laser axis, to recreate the designed angle of incidence for the backlighter targets on NIF. The $\mathrm{CH}-\mathrm{Zn}$ foil was positioned to create a 500um separation distance between the surface of the $\mathrm{Zn}$ foil and the pinhole. The intensity was varied between $1 \times 10^{15}$ and $5 \times 10^{15} \mathrm{~W} / \mathrm{cm}^{2}$ on the $\mathrm{Zn}$ foil and was controlled by changing the number of beams on target. The backlighter performance was characterized using a gold foil/grid target and imaged onto a single strip X-ray framing camera with a magnification of $M=23$. The $\mathrm{Zn}$ spectrum was recorded using the SSC-A SXS streaked spectrometer with a photon energy range of $7.76-12.87 \mathrm{keV}$. The resolution of the system was measured to be $20 \mu \mathrm{m}$ mode, which corresponds to a $20 \%$ transmission in the MTF for all shots. From the spectrometer the X-ray signal was primarily monochromatic in the observed photon energy range for all tested intensities; however there was reduced contrast in the XRFC images for intensities above $4 \times 10^{15} \mathrm{~W} / \mathrm{cm}^{2}$. Peak contrast recorded for the experiment was at intensities around $2.5 \times 10^{15} \mathrm{~W} / \mathrm{cm}^{2}$. From the streaked spectrometer, the $\mathrm{He}_{\alpha}$ lines scale well with the intensity of the beam. The angle of incidence did not have a noticeable effect on the peak $\mathrm{Zn} \mathrm{He}_{\alpha}$ signal or the resolution of the backlighter. The results from this data were used to confirm the backlighter design for NIF experiments and have led to high quality images on NIF.
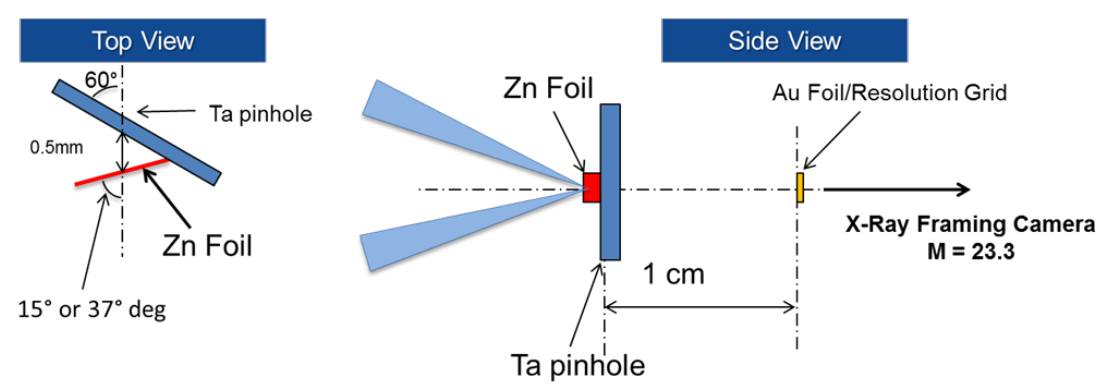

XRFC Image

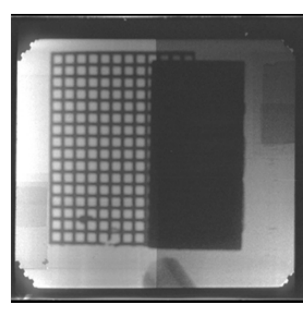

Figure 1) Cartoon of the experimental setup, showing angles of the Ta pinhole substrate and $\mathrm{Zn}$ foil with respect to the laser/imaging axis. Shown on the right is a sample XRFC image of the Au Grid and $\mathrm{Au}$ foil. A $\mathrm{Zn}$ filter covered half the image and 2 sets of 4 and $8 \mathrm{Cu}$ filter steps are also present in the corners. 


\section{X-ray Area Backlighter Development (PI: K. Baker)}

A campaign of six shots was carried out on the OMEGA laser to measure the conversion efficiency and uniformity of zinc backlighters. A common platform was used to evaluate the conversion efficiency from $\mathrm{Zn}$ foils with and without a $2.8 \mathrm{~ns}$ prepulse and $\mathrm{ZnO}$ coated aerogels and $\mathrm{ZnO}$ foams. The common platform consisted of a $2 \mathrm{~mm}$ diameter by $2 \mathrm{~mm}$ long tube that was either filled with a low density foam or had two foils glued on the ends of an empty tube. As shown in Fig. 1, the highest overall conversion efficiency came from a 5 um thick $\mathrm{Zn}$ foil target driven with a 2.8 ns prepulse which was $\sim 3 \mathrm{x}$ brighter than the same $\mathrm{Zn}$ foil without a prepulse. The second most efficient target was a pure $\mathrm{ZnO}$ foam, followed by $\mathrm{ZnO}$ coated $\mathrm{SiO}_{2}$ aerogels. The foam targets, which underwent volume ionization, exhibited more uniform radial emission above $1 \mathrm{keV}$ than the foil targets, with or without prepulse. The thinnest $\mathrm{Zn}$ exploding foil target, 1 um thick, stagnated in the middle of the tube, producing a temporally longer $\mathrm{He}_{a}$ emission than the other targets.

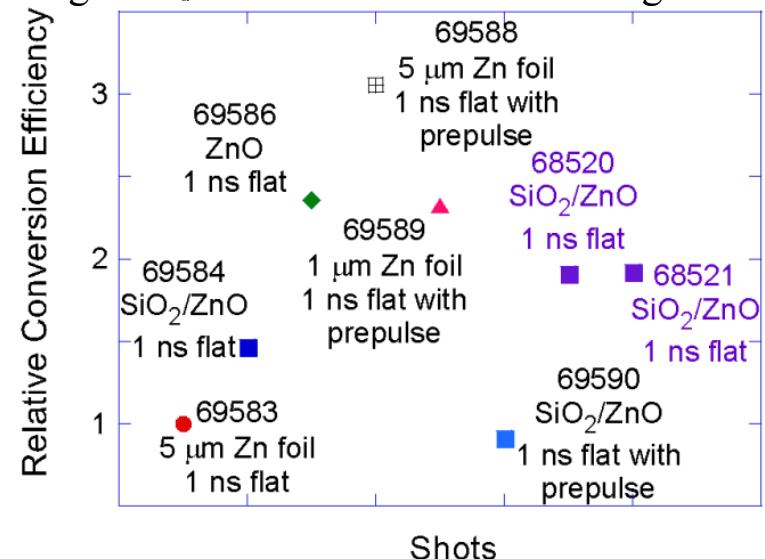

Figure 1 Relative energy conversion efficiency for the various zinc targets.

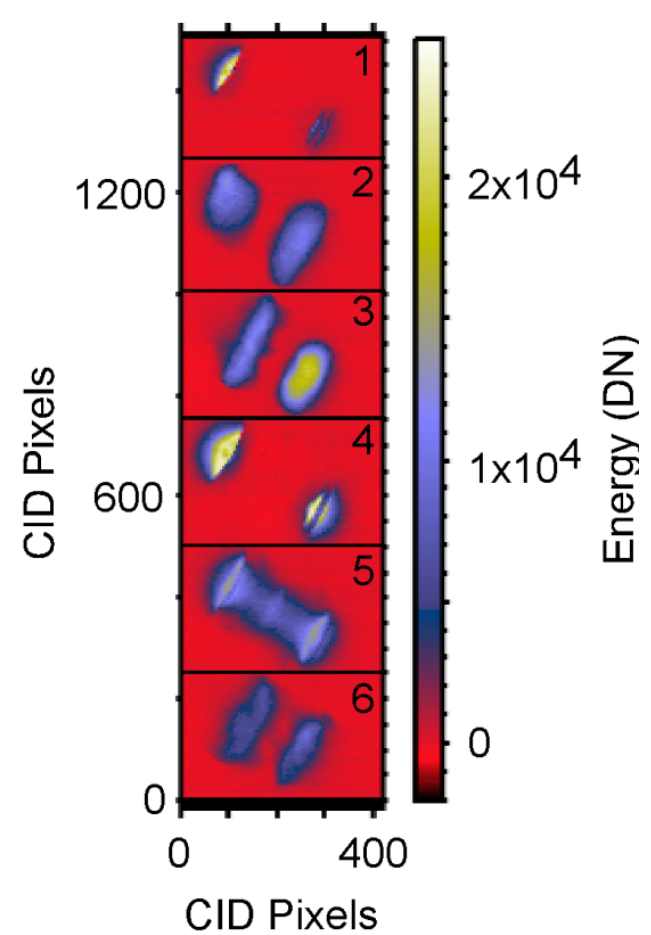

Figure 2 Time-integrated $\mathrm{x}$-ray pinhole images of the various targets showing the increased fluence from the foil targets with prepulse, 4 , and the greatly increased uniformity with the low density foams; 2,3 and 6 . 


\section{Burn Physics}

\section{A. High-resolution measurements of velocity nonuniformities in an alternative ignition capsule ablator material, boron carbide (CAPSEED - PI: P. Celliers)}

Two days of CAPSEED campaigns took place in FY13. These campaigns began initial evaluations of boron carbide $\left(\mathrm{B}_{4} \mathrm{C}\right)$ as an alternate ablator material for NIF ignition target designs. The large fraction of boron in this material makes it a particularly efficient ablator, similar to others such as $\mathrm{CH}$ and $\mathrm{C}$. However, $\mathrm{B}_{4} \mathrm{C}$ undergoes brittle failure when stresses exceed the yield stress, thus it is anticipated that it may have a complicated shock response, similar to diamond. Two CAPSEED campaigns this year provided the initial evaluations of ablator non-uniformities produced in $\mathrm{B}_{4} \mathrm{C}$ under shock.

For the CAPSEED-13A campaign the $\mathrm{B}_{4} \mathrm{C}$ samples were obtained from a commercial source using a sintering process. Characterization of the sample revealed carbon inclusions and voids, and the polished samples incurred large (micron-sized) defects on the surfaces owing to these issues. Attempts to mitigate these problems were made, but the resulting shock quality was severely compromised. The second campaign, CAPSEED-13B, investigated samples that were also created through a sintering process developed at LLNL, but with much lower levels of non-uniformity and voids. Results from the later samples showed a clear improvement, however, the shock front non-uniformities in these samples were still clearly evident in the data as shown in Fig. 1. The velocity spectra of the current $\mathrm{B}_{4} \mathrm{C}$ samples, when compared with high quality Be and GDP ablator samples, are too non-uniform for use in a NIF capsule. Improvements in the fabrication processes to control these non-uniformities are required for further progress with this material. 


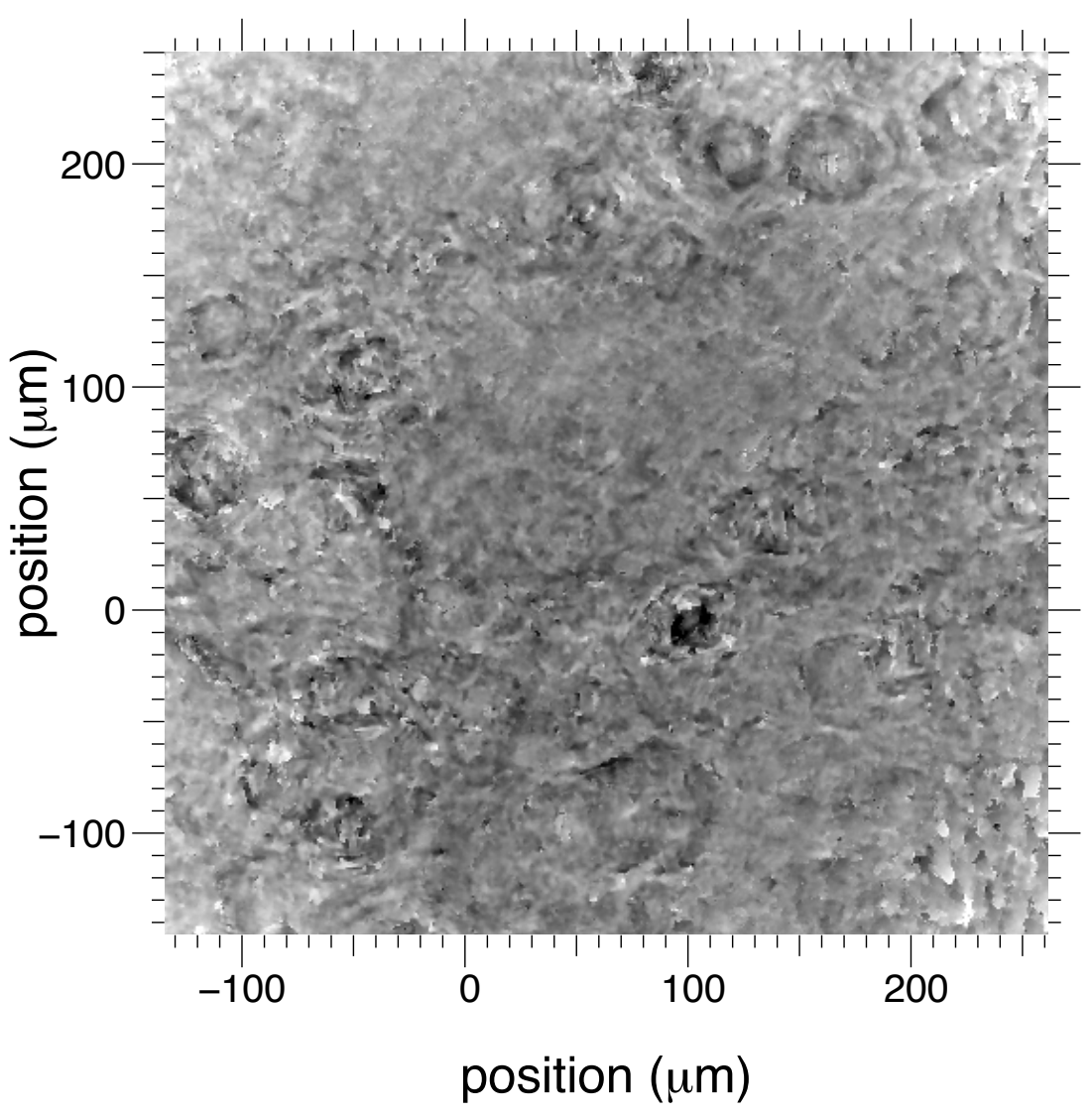

Figure 1: Gray scale representation of the shock front velocity as measured on 69581 , a $\mathrm{B}_{4} \mathrm{C}$ sample shocked to $6.2 \mathrm{Mbar}$. The presence of voids and carbon inclusions distributed throughout the sample resulted in the circular ripple-like disturbances in the velocity pattern. These disturbances produce most of the spectral power in the $10-100 \mu \mathrm{m}$ wavelength range $(0.01-$ $0.1 \mu \mathrm{m}^{-1}$ spatial frequency range). 


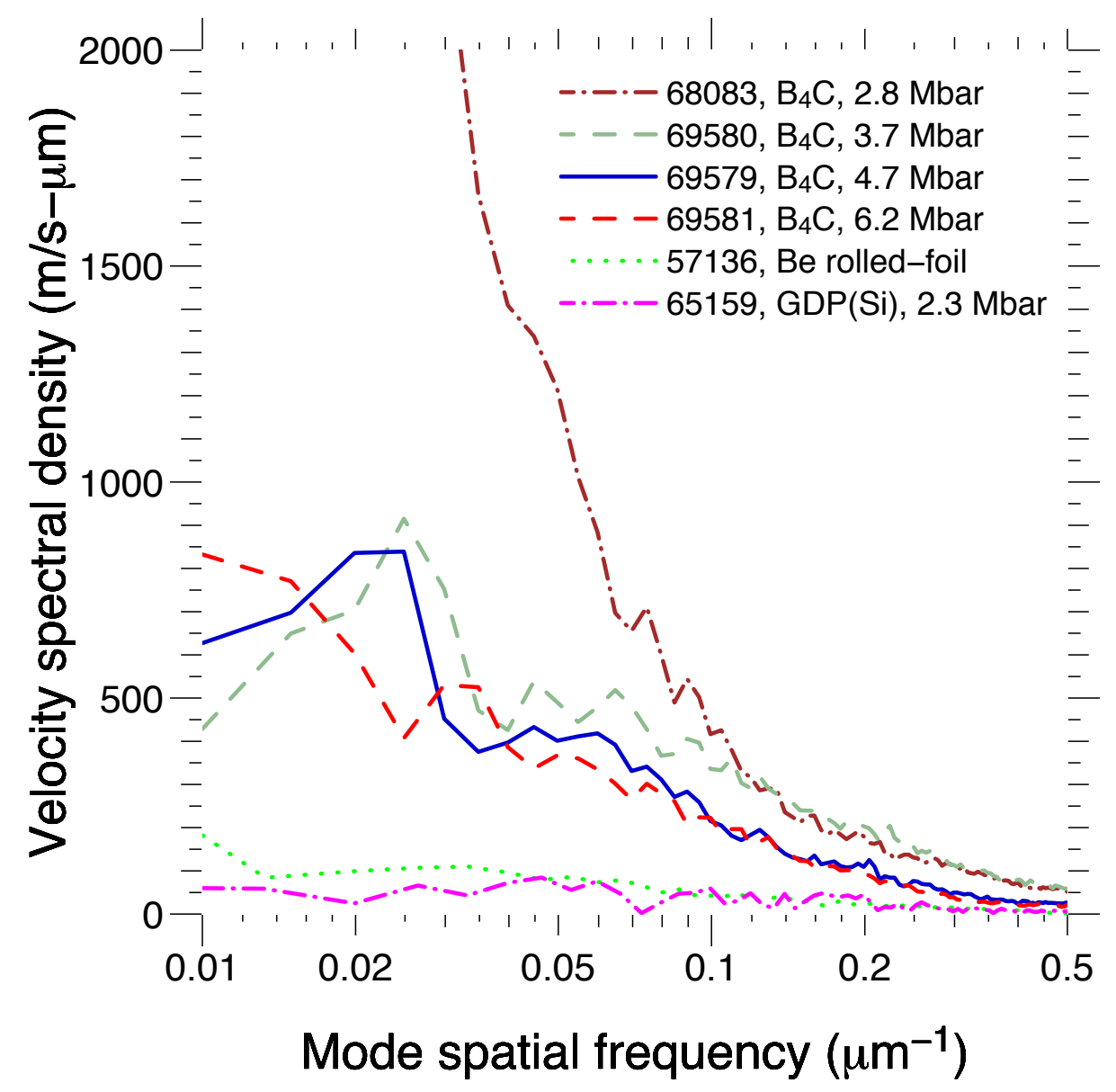

Figure 2: Velocity fluctuation spectra measured for $\mathrm{B}_{4} \mathrm{C}$ from CAPSEED-13A (low-quality sample, 68083), and with improved samples CAPSEED-13B $(69580,69579,69581)$. Fluctuation levels are still a factor of 5 to 10 times too high for use as a NIF ablator. For comparison, results from a NIF-quality Be foil (57136) and silicon-doped glow discharge polymer (GDP) sample (65159) show much lower fluctuation levels. 


\section{B. Ablator Physics: Tests of Beryllium Capsules (PI: D. Casey)}

The goal of the AblatPhys-J-13A OMEGA/EP joint shot day was to demonstrate Be as a high performance ablator for indirect drive ignition experiments on the NIF. These experiments were diagnosed with the OMEGA neutronics suite, and also used EP-driven $\mathrm{Cu}$ K-alpha backlighting observed with the spherical crystal imager. This was the first use of the spherical crystal imager in indirect drive at OMEGA, and excellent data was obtained that resulted in high quality radiographs and neutronics data. The capsules had $600 \mu \mathrm{m}$ diameters with $30 \mu \mathrm{m}$ thick Be walls (where the inner $7.5 \mu \mathrm{m}$ was doped with $4 \% \mathrm{Cu}$ ) and were filled with deuterium gas. GA, who manufactured the capsules, tackled a number of complex target fabrication issues, including: deuterium gas retention, Be polishing, $\mathrm{Cu}$ diffusion, and residual glue from the gas fill hole. Several targets also included thin $0.1 \mu \mathrm{m} \mathrm{W}$ layers on the inner capsule surface to provide radiographic contrast. The capsules were driven using $1.6 \mathrm{~mm}$ diameter by $2.1 \mathrm{~mm}$ long by $50 \mu \mathrm{m}$ thick Au hohlraums irradiated by 40 OMEGA laser beams. These targets performed exceptionally well, providing around $80 \%$ of the neutron yield expected based on simulations. The figure below shows the radiograph geometry, a sample radiograph, and the Be target performance (without $\mathrm{W}$ layer) as indicated by the yield divided by the simulated yield (YOS).
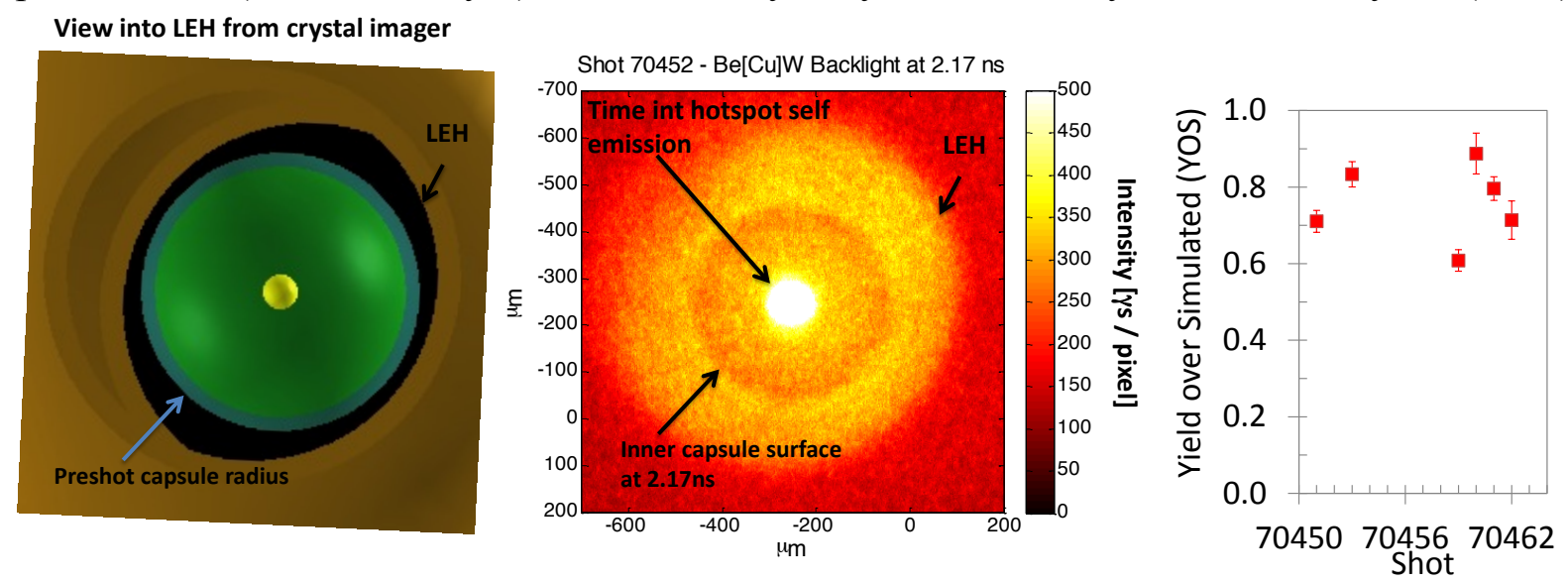


\section{X-Ray Source Development and Application}

\section{A. Solar Cell Electrostatic Discharge (PI: R. Patterson)}

The SolarCell ElectroStatic Discharge campaign is a joint effort between the Naval Research Laboratory, the Defense Threat Reduction Agency, and the X-Ray Source Development team at LLNL. The goal of this campaign is to establish a platform capable of performing X-ray exposure testing on arrays of solar cells ${ }^{1}$, specifically to demonstrate that a sustained arc can be formed between individual cells.

On July 11 2013, the team successfully fielded the time-of-flight Langmuir probe (XLPD) ${ }^{2}$ and active solar cell array (XLPDAC) diagnostics on 10 shots, obtaining excellent data indicative of electrostatic discharge over a range of source-to-cassette distances between $60 \mathrm{~cm}$ and $96 \mathrm{~cm}$. The improvements in robustness and reproducibility of acquired data resulted from modifications to the XLPDAC hardware (Fig. 1), including electrical insulation of the solar cells from the aluminum cassette and improved shielding of previously exposed contacts.

XLPD measurements confirmed the arrival of the source plasma a few $\mu$ s after $\mathrm{x}$-ray loading ${ }^{3}$, consistent with prior measurements of plasma velocities $\sim 15 \mathrm{~cm} / \mu \mathrm{s}$. Fig. 2 shows the solar cell output signals for several shots. The data suggest that the arc formation is not between the cells themselves. The cell biased at $+100 \mathrm{~V}$ and limited to $4 \mathrm{~A}$ max current behaves similarly to an area Langmuir probe, drawing current from the surrounding plasma. The other cell was grounded and not current limited, showing signals consistent with initial photo-ionization of the cover glass and a subsequent decrease in amplitude resulting from neutralization with the plasma. Future experiments will include different or no current limits on the cells and cell geometry variations that allow discrimination between arcing and photoionization effects (geometry independent vs. scaling with area).

${ }^{1}$ P. P. Jenkins, et al., Nuclear Weapons Effects Testing of Solar Cells Using the National Ignition Facility (NIF), 201035 th IEEE Photovoltaic Specialists Conference (PVSC) 002550-3 (2010).

${ }^{2}$ J. R. Patterson, et al., A Langmuir Probe Diagnostic For Use In Inhomogeneous, Time-Varying Plasmas Produced By High Energy Laser Ablation, Review of Scientific Instruments 83, $10 \mathrm{D} 725$ (2012).

${ }^{3}$ K.B. Fournier, et al., Absolute X-Ray Yields From Laser-Irradiated Ge-Doped Low-Density Aerogels, Physics of Plasmas 16, 052703 (2009). 


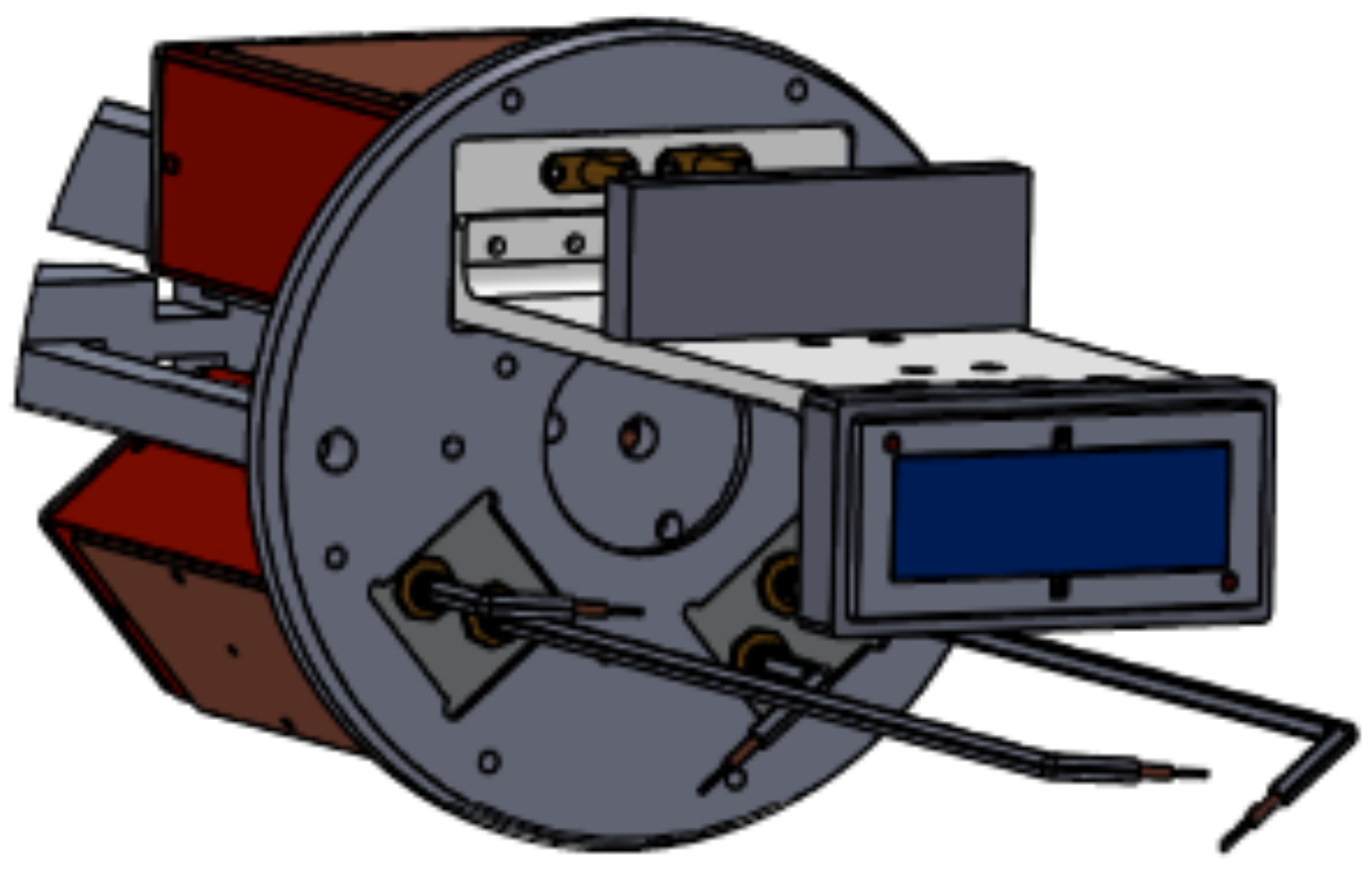

Fig. 1: A CAD model including the modifications made to the XLPDAC. Mounted from the circular aluminum cassette face are the solar cell support bracket (lt. grey) with cells (blue), and two pairs of Langmuir probes beneath. The bias and power circuitry housing (red) is attached to the rear of the cassette. 


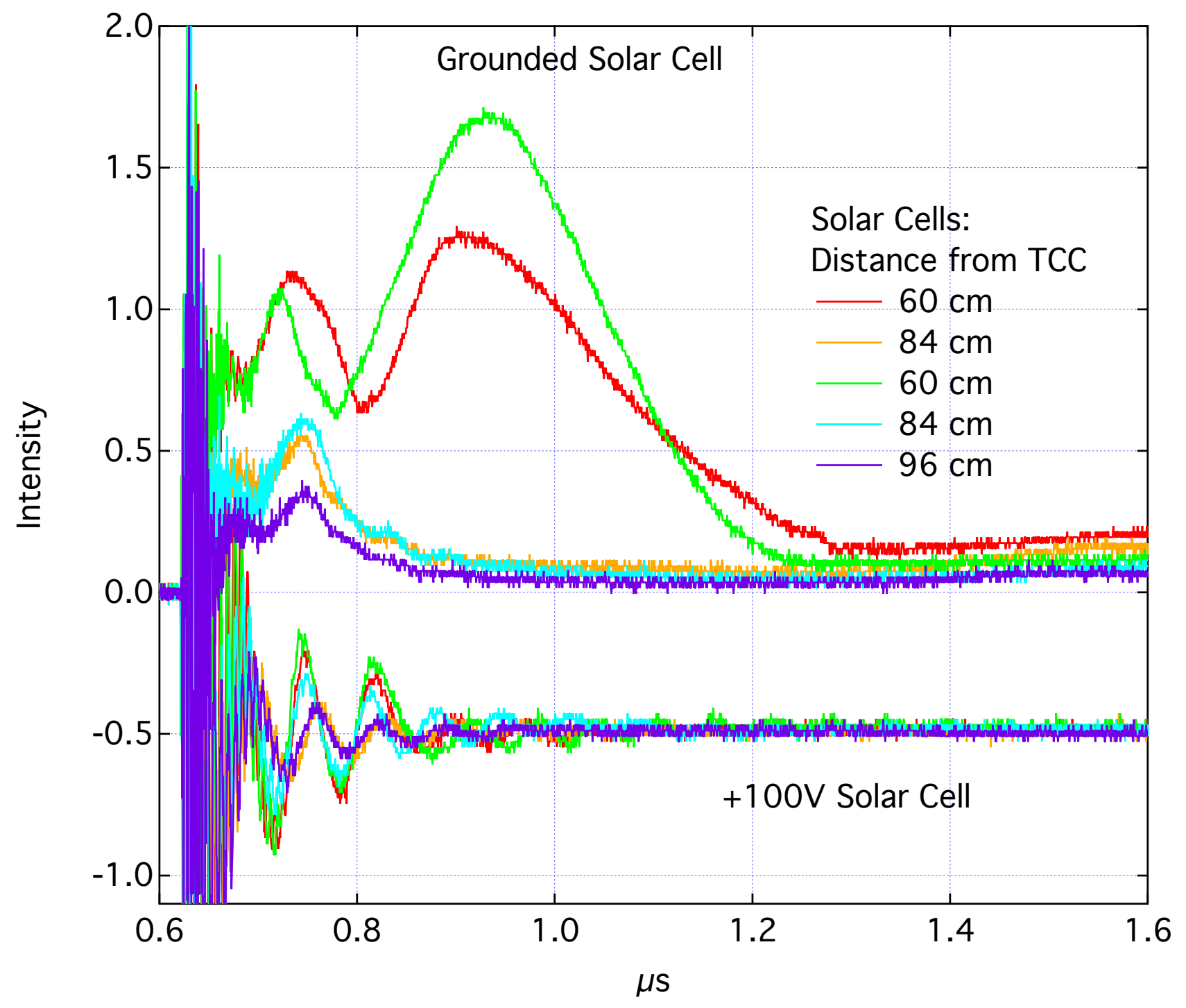

Fig. 2: Voltages recorded from grounded (upper) and $+100 \mathrm{~V}$ biased (lower) solar cells. Signals are from shots with Ge-doped $\mathrm{SiO}_{2}$ aerogel x-ray sources, irradiated with $\sim 20 \mathrm{~kJ}$ in a 1 ns square pulse. 


\section{B. X-ray Source Development with Nanostructured Materials (PI: F. Perez)}

In FY13 the X-Ray Source Development team, funded by the Defense Threat Reduction Agency, created the most efficient Ti-doped foam for generating bright, ns-class X-ray pulses to date [manuscript in preparation]. Two new fabrication techniques achieved record concentrations of Ti emitters (up to 33 atomic $\%$ ) by coating $\mathrm{TiO}_{2}$ on an aerogel or nano-porous scaffold. These foams now demonstrate laserto-X-ray conversion efficiencies above 5\%, comparable to other types of X-ray-source targets such as metallic cavities and pre-exploded foils. The X-rays are generated when 40 beams of the Omega laser irradiate a foam sample (see Fig. 1).

This year, after obtaining X-ray flux and laser-heating propagation measurements in the targets, the team found that the dynamics of the foam heating were not well reproduced by state-of-the-art computer simulations. This discrepancy, under investigation, could be explained by an unexpected role of foam structure, metal-dopant content, the presence of absorbed water, or by effects not included in the simulations. Understanding and mitigation of these effects may lead to new designs and even higher xray efficiencies.

In parallel, new types of foams are being investigated. The present limitation is that most of the foam is made of elements lighter than $\mathrm{Ti}$ ( $\mathrm{Si}$ and $\mathrm{O}$ ), which channel energy out of the system. Cu-based foams, containing only trace amounts of light elements, were tested this year for the first time. Being still at an early stage of development, the current results will serve as a reference point for next year's ultra-lowdensity $\left(<10 \mathrm{mg} / \mathrm{cm}^{3}\right)$, almost pure-Cu foams.

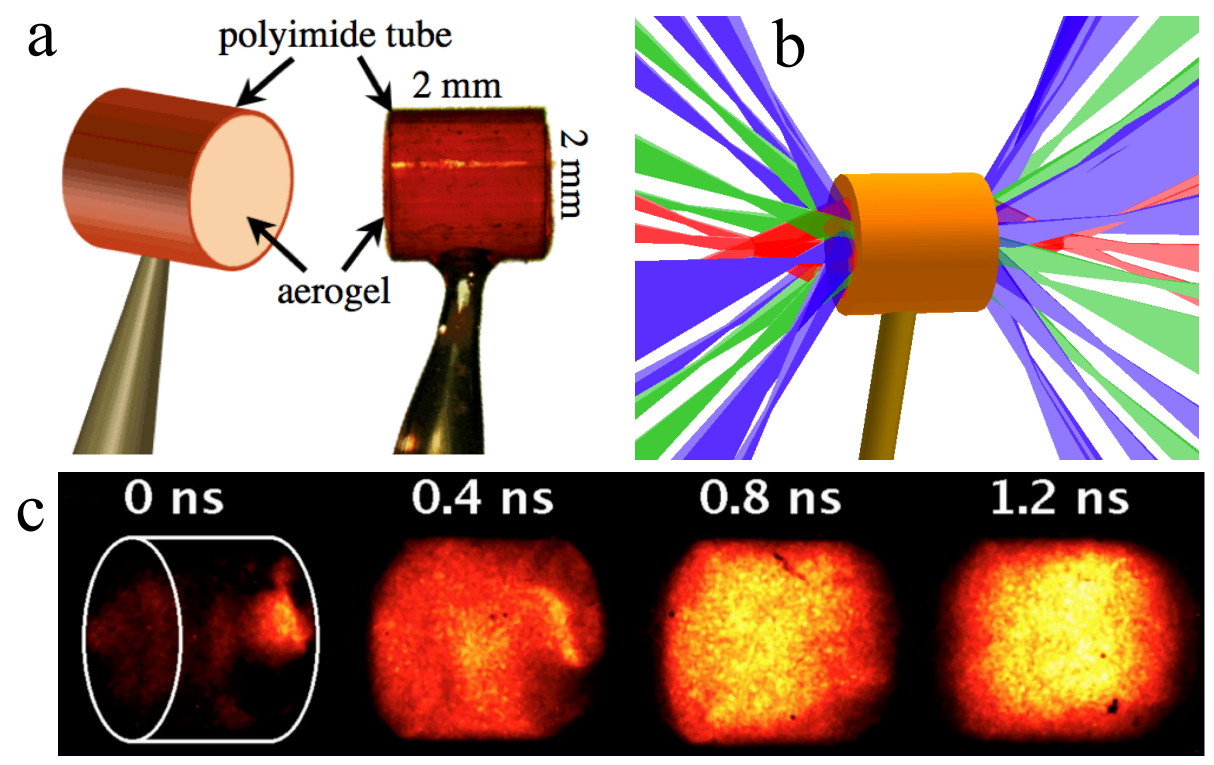

Fig. 9: (a) foam sample held in a plastic tube. (b) laser irradiation pattern of the Omega laser. (c) $X$-ray emission images at different times relative to the beginning of the interaction. 